\title{
MULTISCALE SCANNING IN INVERSE PROBLEMS
}

\author{
By Katharina ProksCH*, Frank Werner ${ }^{\dagger}$ And Axel MunK ${ }^{*, 2}$ \\ University of Göttingen* and Max Planck Institute for Biophysical Chemistry ${ }^{\dagger}$
}

In this paper, we propose a multiscale scanning method to determine active components of a quantity $f$ w.r.t. a dictionary $\mathcal{U}$ from observations $Y$ in an inverse regression model $Y=T f+\xi$ with linear operator $T$ and general random error $\xi$. To this end, we provide uniform confidence statements for the coefficients $\langle\varphi, f\rangle, \varphi \in \mathcal{U}$, under the assumption that $\left(T^{*}\right)^{-1}(\mathcal{U})$ is of wavelet-type. Based on this, we obtain a multiple test that allows to identify the active components of $\mathcal{U}$, that is, $\langle f, \varphi\rangle \neq 0, \varphi \in \mathcal{U}$, at controlled, familywise error rate. Our results rely on a Gaussian approximation of the underlying multiscale statistic with a novel scale penalty adapted to the ill-posedness of the problem. The scale penalty furthermore ensures convergence of the statistic's distribution towards a Gumbel limit under reasonable assumptions. The important special cases of tomography and deconvolution are discussed in detail. Further, the regression case, when $T=$ id and the dictionary consists of moving windows of various sizes (scales), is included, generalizing previous results for this setting. We show that our method obeys an oracle optimality, that is, it attains the same asymptotic power as a single-scale testing procedure at the correct scale. Simulations support our theory and we illustrate the potential of the method as an inferential tool for imaging. As a particular application, we discuss super-resolution microscopy and analyze experimental STED data to locate single DNA origami.

1. Introduction. Suppose we have access to observations $Y_{\mathbf{j}}$ which are linked to an unknown quantity $f \in \mathbb{H}_{1}$ via the inverse regression model

$$
Y_{\mathbf{j}}=T f\left(\mathbf{x}_{\mathbf{j}}\right)+\xi_{\mathbf{j}}, \quad \mathbf{j} \in I_{n}^{d}:=\{1, \ldots, n\}^{d}, d \in \mathbb{N} .
$$

Here, $T: \mathbb{H}_{1} \rightarrow \mathbb{H}_{2} \subset C[0,1]^{d}$ is a bounded linear operator acting between proper Hilbert spaces $\mathbb{H}_{1}$ and $\mathbb{H}_{2}$. In model (1), $n$ stands for the level of discretization such that, more rigorously, the model reads $Y_{\mathbf{j}, n}=T f\left(\mathbf{x}_{\mathbf{j}, n}\right)+\xi_{\mathbf{j}, n}$ with triangular schemes of sampling points $\mathbf{x}_{\mathbf{j}}=\mathbf{x}_{\mathbf{j}, n}$ in the $d$-cube $[0,1]^{d}$ and independent, centered but not necessarily identically distributed random variables $\xi_{\mathbf{j}}=\xi_{\mathbf{j}, n}, \mathbf{j} \in I_{n}^{d}$. For ease of notation, this dependence on $n$ is suppressed whenever it is not relevant. Here and throughout the paper, bold print letters and numbers denote vectors and multi-indices, whereas scalars are printed in regular type face.

Received June 2017; revised October 2017.

${ }^{1}$ Supported by the German Research Foundation DFG through subproject A07 of CRC 755.

${ }^{2}$ Funding through the VW foundation is also gratefully acknowledged.

MSC2010 subject classifications. Primary 62G10; secondary 62G15, 62G20, $62 \mathrm{G} 32$.

Key words and phrases. Multiscale analysis, scan statistic, ill-posed problem, deconvolution, super-resolution, Gumbel extreme value limit. 
Models of the kind (1) underly a plenitude of applied problems varying from astrophysics and tomography to cell biology [see, e.g., O'Sullivan (1986), Bertero et al. (2009)] and have received considerable interest in the statistical literature. Most of research targets (regularized) estimation of $f$ and associated theory. An early approach for estimation is based on a singular value decomposition (SVD) of the operator, where $f$ is expanded in a series of eigenfunctions of $T^{*} T$ [see, e.g., Mair and Ruymgaart (1996), Johnstone et al. (2004), Cavalier and Golubev (2006), Bissantz et al. (2007), Kerkyacharian et al. (2010), Johnstone and Paul (2014), Albani et al. (2016)]. Given a proper choice of the regularization parameter, SVD-based estimators are well known to be minimax optimal [Johnstone and Silverman (1991)]. Adaptive estimation in this context was studied, for example, by Cavalier et al. (2003), Chernousova and Golubev (2014), Goldenshluger (1999), Tsybakov (2000). Since in SVD-based estimation the basis for the expansion is entirely defined by the operator, as an alternative, wavelet-based methods which incorporate the properties of the function of interest have also been frequently employed. Examples are wavelet-vaguelette [Donoho (1995)] and vaguelette-wavelet methods [Abramovich and Silverman (1998)], where $f$ and $K f$ are expanded in a wavelet and vaguelette basis or vice versa, and the coefficients are estimated by proper thresholding. This allows for a natural adaptation to the local smoothness of the unknown function [see, e.g., Cavalier and Tsybakov (2002)]. Related to this, Cohen, Hoffmann and Reiß (2004) proposed an adaptive estimator based on a combination of linear Galerkin projection methods and adaptive wavelet thresholding. Besides of these selective references a vast amount of work has been devoted to recovery of $f$ during the last decades and the common ground of all these works is that the ill-posedness of an inverse problem usually only gives poor (minimax) rates for estimation and makes full recovery of $f$ a very difficult problem in general [in the setup of (1) see, e.g., Willer (2009), or for deconvolution, see, e.g., the monograph by Meister (2009) and the references given there].

A possibility to deal with this intrinsic difficulty is to relax the ambitious goal of recovering the entire function $f$. Indeed, in many applications, only certain properties or aspects of $f$ are of primary interest and a full, precise reconstruction is not necessary any more. Examples of practical relevance are the detection and localization of "hot spots" in astrophysical image analysis [Friedenberg and Genovese (2013)], functional magnetic resonance imaging [Schwartzman, Dougherty and Taylor (2008)], nondestructive testing [Kazantsev et al. (2002)], and image deformation in microscopy [Bissantz et al. (2009)], to mention a few. For a theoretical account in deconvolution, see Butucea and Comte (2009). In a similar spirit, the detection of certain geometric shapes in image analysis has been studied by Genovese et al. (2012), but the authors do not take into account the underlying inverse problem. All these issues can be treated by means of statistical testing, presumably a simpler task than estimation.

In contrast to estimation, hypothesis testing in inverse problems has been investigated much less, early references are Butucea (2007), Holzmann, Bissantz and 
Munk (2007). Ingster, Sapatinas and Suslina (2012) treat the problem of testing $f=0$ against $f \in \Theta_{q}(r)$ where $\Theta_{q}(r)$ is a suitable smoothness class restricted to $\|f\| \geq r$ by means of the classical minimax testing approach [see, e.g., the series of papers by Ingster (1993)]. Also Laurent, Loubes and Marteau $(2011,2012)$ follow this path and investigate the differences and commonalities of testing in the image space $(T f=0)$ and the preimage space $(f=0)$. The authors prove that in several situations it does not matter if first $f$ is approximately reconstructed using an SVD-based regularization method and then tested to be 0 , or if $T f$ is directly tested to be 0; see also Holzmann, Bissantz and Munk (2007) for a similar observation. More precisely, minimax testing procedures for one of these problems are also minimax for the rephrased problem and the asymptotic detection boundary for both testing problems coincides. For related results in the multivariate setting or for more general regularization schemes, see Ingster, Laurent and Marteau (2014), Marteau and Mathé (2014). In contrast to the problem treated here, in all these studies only "global" features of the full signal are investigated, such as testing that the full signal is zero, and no simultaneous inference on sub-structures of the signal is targeted. In fact, this is a much more challenging task in an inverse problems setup and it turns out also to be substantially different to the corresponding direct testing problem of "hot spot" detection. This will be the topic of this paper.

In direct problems [ $T=\mathrm{id}$ in (1)], finding relevant sub-structures, such as the detection of regions of activity, is of "scanning-type", which means that it can be reformulated as a (multiple) testing problem for structures on the grid $I_{n}^{d}$ in (1) and scanning-type procedures can be employed. These have received much attention in the literature over the past decades. Walther (2010) considers the two-dimensional problem of detecting spatial clusters in the Bernoulli model by scanning with rectangular windows of varying sizes, see also Kabluchko (2011), Butucea and Ingster (2013) and Sharpnack and Arias-Castro (2016) for results in a Gaussian setting. In a similar spirit, scan statistics have been employed in the context of multiscale inference about higher order qualitative characteristics such as modes of a density [see Dümbgen and Walther (2008), Rufibach and Walther (2010), Li et al. (2016), Eckle et al. (2018)].

However, in an inverse problem as in (1), it is not obvious how to perform statistically efficient "scanning" because local properties of $f$ may propagate in a nonlocal manner into $T f$. If, for example, $f$ is a function on $[0,1]^{d}$ and we want to infer on the support of $f$, we find that despite the fact that globally testing $f \equiv 0$ is equivalent to testing $T f \equiv 0$, this is not true for localized tests on regions $B \subset[0,1]^{d}$ we are interested in here. This is due to the fact that $(T f)_{\left.\right|_{B}}$ is not necessarily related to $f_{\left.\right|_{B}}$ only. Indeed, we will see that reducing this problem to the image domain $\mathbb{H}_{2}$, that is, simultaneously testing $H_{B}:(T f)_{\left.\right|_{B}} \equiv 0$ against $K_{B}:(T f)_{\left.\right|_{B}}>0$ cannot lead to a competitive procedure as it does not take into account the propagation of (multiscale) features of $f$ by $T$ [cf. Figure 2(f)]. Instead, it becomes necessary to employ probe functionals $\varphi_{i}=\varphi_{i, n}$ (again dependent on the discretization level $n$, but this dependence will be suppressed whenever not 
relevant below), which are compatible with the operator $T$, and hence allow for transportation of "local" information from $T f$ back to $\left\langle f, \varphi_{i}\right\rangle$. If the probe functionals $\varphi_{i}$ are chosen properly, the values $\left\langle f, \varphi_{i}\right\rangle$ hold information about "local" features of $f$, for example, in the form of a wavelet-type analysis; see also Eckle, Bissantz and Dette (2017), Schmidt-Hieber, Munk and Dümbgen (2013), who infer on shape characteristics in i.i.d. density deconvolution. Arias-Castro, Donoho and Huo (2005) propose a scanning procedure based on a multiscale dictionary of beamlets that allows to detect line segments hidden in a noisy image, however, not in an inverse problems context.

The problem we consider in our paper is as follows: Given model (1) and an associated sequence of dictionaries

$$
\mathcal{U}=\mathcal{U}_{n}=\left\{\varphi_{1, n}, \ldots, \varphi_{N(n), n}\right\} \subset R\left(T^{*}\right),
$$

of cardinality $N=N(n) \rightarrow \infty$ as $n \rightarrow \infty$, we provide a sequence of multiple tests ("scanning") for the associated sequence of multiple testing problems

$\left(H_{J, n}\right) \quad\left\langle f, \varphi_{i, n}\right\rangle=0 \quad$ for all $i \in J$

versus

$\left(K_{J, n}\right) \quad \exists i \in J \quad$ such that $\left\langle\varphi_{i, n}, f\right\rangle>0$,

simultaneously over all subsets $J \subset I_{N(n)}=:\{1, \ldots, N(n)\}$. It is clear that the structure of the testing problem stays the same if $\cdot>0$ in $\left(K_{J, n}\right)$ is replaced by $\cdot<0$ or $|\cdot| \neq 0$, hence we restrict ourselves to $\left(K_{J, n}\right)$ in the following. Moreover, it is also clear that as $n \rightarrow \infty$, there is a detection boundary, given by a sequence $\left(\mu_{i, n}\right)_{i \in \mathbb{N}}$, dividing the space of all signals into the asymptotically detectable region and the nondetectable region such that $\cdot>0$ will be replaced by $\cdot>\mu_{i, n}$ later on.

The underlying idea of the present paper is to provide for each local testing problem a local test which detects those coefficients $\left\langle f, \varphi_{i, n}\right\rangle, i \in J$, which are strong enough, and hence by performing all these tests simultaneously, we expect to (asymptotically) detect all positive coefficients above the detection boundary. If $f$ admits a sparse representation w.r.t. $\mathcal{U}$, this is $f \approx \sum c_{i, n} \varphi_{i, n}$ with $\|c\|_{0}$ small, then the simultaneous testing problem $H_{J, n}$ against $K_{J, n}, J \subset I_{N}(n)$ allows to detect exactly those $i$ with $c_{i, n}>0$. However, we emphasize that sparsity of $f$ w.r.t. $\mathcal{U}$ is not required or assumed here.

With this choice of a sequence of multiple tests, we will not simply control the error of a wrong rejection of $f \equiv 0$, rather we control the family-wise error rate (FWER) of making any wrong decision; cf. Dickhaus (2014), Definition 1.2. Mathematically, our test is a level- $\alpha$-test for the simultaneous testing problem $H_{J, n}$ against $K_{J, n}, J \subset I_{N}(n)$, that is, it guarantees that

$$
\sup _{J: J \subset I_{N(n)}} \mathbb{P}_{H_{J, n}}[\text { "at least one (wrong) rejection in } J "] \leq \alpha+o(1),
$$


as $n$, and hence $N(n) \rightarrow \infty$. Consequently, all rejections (i.e., decisions for signal strength $>0$ ) will be made at a uniform error control, no matter what the underlying configuration of $\left\langle f, \varphi_{i, n}\right\rangle$ 's is.

Fundamental to our simultaneous scanning procedure are uniform confidence statements for the coefficients $\left\langle f, \varphi_{i, n}\right\rangle, i \in I_{N(n)}$ in the inverse regression model (1). Conceptually related, Nickl and Reiß (2012) and Söhl and Trabs (2012) provide uniform Donsker-type results in the context of i.i.d. deconvolution for singlescale contrasts $\langle f, \varphi\rangle$. As one particular example the results of the latter authors can be used to derive uniform statements with respect to both the regularization parameter $h$ (which plays the role of a scale parameter) and variable location $t$ via the functionals $\left\langle I_{(-\infty, 0]}(\cdot-t), \widehat{f_{h}}\right\rangle=: \widehat{F}_{h}(t)$ as estimators of the distribution function $F$, where $\widehat{f}_{h}$ is a deconvolution estimator of the density $f$. We consider dictionaries which are different in that they are closely related to estimating the regression function $f$ in (1) (which would correspond to estimating $f$, not $F$, in their model), where uniform control with respect to the scale parameter requires the use of very different techniques.

Multiscale approaches have also been discussed in the Bayesian literature [see, e.g., Castillo and Nickl (2014), Ray (2017)], but not in the inverse problems setup. Even though it seems promising to exploit Gaussian approximations based on posterior distributions as in Castillo and Nickl (2014), this leads to additional difficulties in our general setup as typically conjugacy is lost in inverse regression problems if the likelihood and/or the prior are non-Gaussian. Consequently, sampling from the posterior becomes a computationally involved large-scale problem. Nevertheless, we stress that in principle recent developments for nonparametric Bayesian credible sets [see, e.g., Knapik, van der Vaart and van Zanten (2011), Ray (2013)] can offer an alternative route to the present methodology.

1.1. Multiscale Inverse SCAnning Test: MISCAT. As we have assumed that $\varphi_{i, n} \in \mathcal{R}\left(T^{*}\right)$ for all $i \in I_{N(n)}$, there exists a sequence of dictionaries $\mathcal{W}=\mathcal{W}_{n}=$ $\left\{\Phi_{i, n} \mid i \in I_{N(n)}\right\} \subset \mathbb{H}_{2}$ such that $\varphi_{i, n}=T^{*} \Phi_{i, n}$. In the following, we will assume that $\mathcal{W}$ obeys a certain wavelet-type structure, that is, for each $i \in I_{N(n)}$ there is an associated scale $\mathbf{h}_{i, n}=\left(h_{i, n, 1}, \ldots, h_{i, n, d}\right)^{T} \in(0,1]^{d}$ and an associated translation $\mathbf{t}_{i, n} \in\left[\mathbf{h}_{i, n}, \mathbf{1}\right]$. The products $\mathbf{h}_{i, n}^{\mathbf{1}}:=h_{i, n, 1} \cdot \ldots \cdot h_{i, n, d}$ will be referred to as sizes of scales. In contrast to the direct problem $(T=\mathrm{id})$, in an inverse problem the condition $\varphi_{i}=T^{*} \Phi_{i}$ implies a nonstandard scaling of the $\Phi_{i}$ 's which can be chosen to depend only on $\mathbf{h}_{i}$ and not on $\mathbf{t}_{i}$ in many cases. To highlight this scaling property, with a slight abuse of notation, we will also introduce a sequence of dictionary functions $\Phi_{\mathbf{h}_{i, n}}$ and assume that $\mathcal{W}_{n}$ is as follows:

$$
\mathcal{W}_{n}=\left\{\Phi_{i, n}(\mathbf{z}):=\Phi_{\mathbf{h}_{i, n}}\left(\frac{\mathbf{t}_{i, n}-\mathbf{z}}{\mathbf{h}_{i, n}}\right) \mid \operatorname{supp}\left(\Phi_{\mathbf{h}_{i, n}}\right) \subset[0,1]^{d}, i \in I_{N(n)}\right\} .
$$

Here and in the following, division of a vector by a vector is meant componentwise. All quantities depend on $n$, and this dependence is suppressed in the following, for example, we write $\Phi_{i}$ instead of $\Phi_{i, n}$. Note that if $\Phi_{\mathbf{h}_{i}} \equiv \Phi$ for all $i \in I_{N}$, 
then the dictionary (4) is a wavelet dictionary in the classical sense, which is appropriate for direct regression problems, that is, $T=\mathrm{id}$ in (1) [see, e.g., Arias-Castro, Donoho and Huo (2005)]. For our asymptotic results, we will further assume that the normed functions $\Phi_{\mathbf{h}_{i}} /\left\|\Phi_{\mathbf{h}_{i}}\right\|$ satisfy an average Hölder condition; see (AHC) or (15) below. Such conditions are satisfied for many important operators $T$ such as the Radon transform (see Section 3.1) and convolution operators (see Section 3.2).

To construct a level- $\alpha$-test for simultaneously testing $H_{J, n}$ against $K_{J, n}, J \subset I_{N}$ we can now employ

$$
\left\langle f, \varphi_{i}\right\rangle_{\mathbb{H}_{1}}=\left\langle T f, \Phi_{i}\right\rangle_{\mathbb{H}_{2}}
$$

to estimate the local coefficients $\left\langle f, \varphi_{i}\right\rangle$ by their empirical counterparts

$$
\left\langle Y, \Phi_{i}\right\rangle_{n}:=\frac{1}{n^{d}} \sum_{\mathbf{j} \in I_{n}^{d}} Y_{\mathbf{j}} \Phi_{i}\left(\mathbf{x}_{\mathbf{j}}\right)
$$

cf. (4) for the definition of $\Phi_{i}$ and see Section 3 for details. MISCAT combines these local statistics to a global level- $\alpha$-test in the sense of (3) no matter what the (local) dependency structure is. To this end, we take the maximum of the local test statistics, yielding a multiple "dictionary scanning" test statistic of the form

$$
\mathcal{S}(Y):=\max _{i \in I_{N}} S(Y, i) \quad \text { with } S(Y, i):=\omega_{i}\left(\frac{\left\langle Y, \Phi_{i}\right\rangle_{n}}{\sigma_{i}}-\omega_{i}\right),
$$

where $\sigma_{i}^{2}:=\operatorname{Var}\left[\left\langle Y, \Phi_{i}\right\rangle_{n}\right]$ depend on the variances $\sigma^{2}(\mathbf{j})$ of the errors $\xi_{\mathbf{j}}$, which are unknown in general. For simplicity, all results will be stated with known $\sigma_{i}^{2}$, as all results remain valid if the unknown ones are replaced by appropriate estimates (see Remark 3). The weights

$$
\omega_{i}=\omega_{\mathbf{h}_{i}}\left(K, C_{d}\right)=\sqrt{2 \log \left(K / \mathbf{h}_{i}^{\mathbf{1}}\right)}+C_{d} \frac{\log \left(\sqrt{2 \log \left(K / \mathbf{h}_{i}^{\mathbf{1}}\right)}\right)}{\sqrt{2 \log \left(K / \mathbf{h}_{i}^{\mathbf{1}}\right)}}
$$

provide a proper scale calibration (see Section 2) if $K / \mathbf{h}_{i} \geq \sqrt{e}$ for all $i \in I_{N}$. Since for all results $\max _{i \in I_{N}} \mathbf{h}_{i} \rightarrow \mathbf{0}$, this is satisfied for any fixed $K>0$ if $n$ is large enough and we may assume throughout this paper, without loss of generality, that $\min _{i \in I_{N}} K / \mathbf{h}_{i}^{\mathbf{1}} \geq \sqrt{e}$. In this sense, our results hold for any constant $K>0$, however, in many situations $K$ can be chosen such that the weak limit of $\mathcal{S}(Y)$ in (7) is a standard Gumbel distribution [see Remark 2(c) and Theorems 3 and 5]. $C_{d}$ is an explicit constant only depending on the dimension, the system of scales considered and the degree of $L^{2}$-smoothness of $\Phi_{\mathbf{h}_{i}}$ [see Theorem 1 and Remark 2(b)]. Our scale balancing (8) is in line with Dümbgen and Spokoiny (2001) and others (but notably different as explained in detail below), who pointed out that, in a multiscale setting, some elements of the dictionary may dominate the behavior of the maximum of a scanning statistic and it is most important to balance 
all local tests on the different scales in order to obtain good overall power, that is, a scale dependent correction is necessary.

MISCAT now selects all probe functionals $\Phi_{i, n}$ as "active", where $\mathcal{S}(Y, i)$ is above a certain (universal) threshold, which guarantees (3), to be specified now. To this end, notice that in (3) we have

$$
\sup _{J: J \subset I_{N}} \mathbb{P}_{H_{J, n}}[" \exists \text { rejection in } J "] \leq \mathbb{P}_{0}\left[" \exists \text { rejection in } I_{N(n)} "\right],
$$

where $\mathbb{P}_{0}=\mathbb{P}_{0, n}=\mathbb{P}_{H_{I_{N(n)}, n}}$, corresponding to $f \perp \mathcal{U}_{n}$. The reason for this is that the chance of a false positive among a selection of possible false positives is highest if this selection is as large as possible and all positives are false. Therefore, in order to control the FWER, we only need a universal global threshold $q_{1-\alpha}$ such that $\mathbb{P}_{0}\left[\mathcal{S}(Y)>q_{1-\alpha}\right] \leq \alpha$. To obtain this universal threshold $q_{1-\alpha}$, we will determine the $\mathbb{P}_{0}$-limiting distribution of $\mathcal{S}(Y)$ under a general moment condition including many practically relevant models. Theorem 1(a) in Section 2 provides a distribution-free (i.e., independent of any unknown quantities such as $f$ ) limit, which is obtained as an almost surely bounded Gaussian approximation for the scan statistic (7) by replacing the errors by a standard Brownian sheet $W$, that is,

$$
\mathcal{S}(W):=\max _{i \in I_{N}} S(W, i) \quad \text { with } S(W, i):=\omega_{i}\left(\frac{\left|\int \Phi_{i}(\mathbf{z}) \mathrm{d} W_{\mathbf{z}}\right|}{\left\|\Phi_{i}\right\|_{2}}-\omega_{i}\right) .
$$

Since $\mathcal{S}(W)$ does not depend on any unknown quantities, it can be used to simulate $q_{1-\alpha}$. Exploiting the specific and new choice of calibration in (8) we will furthermore show in Theorem 1(b) that $\mathcal{S}(Y)$ convergences in distribution towards a Gumbel limit for a wide-range of dictionary functions $\Phi_{i}$. As $\mathcal{S}(Y)$ can be seen as a maximum over extreme value statistics of different scales, it follows that the contributions of the different scales are balanced in an ideal way. This result is remarkable, as it provides a general recipe how to calibrate multiscale statistics depending on the degree of smoothness of the probe functionals $\Phi_{i}$ and the system of scales considered. To the best of our knowledge, this is new even in $d=1$, and in addition, it generalizes results by Sharpnack and Arias-Castro (2016) to other systems than rectangular scanning (see Remark 2), and to inverse problems and nonGaussian errors. Note that the calibration proposed by Dümbgen and Spokoiny (2001) for direct regression problems [which is frequently employed in multiscale procedures, see, e.g., Eckle, Bissantz and Dette (2017), Rohde (2008), SchmidtHieber, Munk and Dümbgen (2013), Walther (2010)] is tailored to a continuous observation setting in which all scales within a range $(0, a], a \in \mathbb{R}^{+}$are considered. If this calibration is used in a discrete setting like (1), the overall test-statistic converges to a degenerate limit, since the largest scale $h_{\max }$ has to satisfy $h_{\max } \rightarrow 0$ as $n \rightarrow \infty$, otherwise the finite sample approximations do not converge to their continuous counterparts. Therefore, we propose a different scale calibration which also takes into account the ill-posedness and yields a proper weak limit in many of such cases. 
The approximation in (10) requires a coupling technique to replace the observation errors by i.i.d. Gaussian random variables. To this end, we do not make use of strong approximations by KMT-like constructions [see Komlós, Major and Tusnády (1975) for the classical KMT results and, e.g., Rio (1993) or Dedecker, Merlevède and Rio (2014) for generalizations] as, for instance, Schmidt-Hieber, Munk and Dümbgen (2013) in the univariate case, $d=1$, but we take a different route and employ a coupling of the supremum based on recent results by Chernozhukov, Chetverikov and Kato (2014). Doing so, we can prove the approximation in (10) to hold for a much larger range of scales.

A major benefit of MISCAT is its wide range of applicability and its multiscale detection power. Given the operator $T$, one chooses a dictionary $\mathcal{U}$ of probe functionals as in (2) such that $\mathcal{W}$ is of the form (4). We will demonstrate this for the case of $T$ being the Radon transform in Section 3.1 and for $T$ being a convolution operator in Section 3.2. For the latter situation, we will also discuss an optimal choice of the probe functionals $\varphi_{i}$. Once the dictionaries $\mathcal{U}$ and $\mathcal{W}$ have been obtained, the quantiles $q_{1-\alpha}$ from the Gaussian approximation (10) or its finite sample analogues can be simulated. As it is well known that convergence towards the Gumbel limit is extremely slow, it is beneficial that for deconvolution we find that the limit only depends on the degree of smoothness (see Theorem 4), and hence the finite sample distribution can be pre-simulated in a universal manner.

We will show in Section 2.4 that the power of MISCAT asymptotically coincides with the power of a single-scale oracle test which knows the correct size of the unknown object beforehand. More generally, if prior scale information is available, our method can be adapted immediately to this situation by restricting (7) to this subset, which mat lead to different calibration constants in (8) [see Remark 2(b)]. This will further increase detection power in finite sample situations.

\subsection{MISCAT in action: Locating fluorescent markers in STED super-resolution} microscopy. In Section 3.2, we specify and refine our results to deconvolution which is applied to a data example from nanobiophotonics in Section 4.2 which we briefly review in the following. Suppose that the operator $T$ is a convolution operator having a kernel $k$ such that

$$
(T f)(\mathbf{y})=(k * f)(\mathbf{y})=\int_{\mathbb{R}^{d}} k(\mathbf{x}-\mathbf{y}) f(\mathbf{y}) \mathrm{d} \mathbf{y} .
$$

In our subsequent application, the convolution kernel $k$ corresponds to the point spread function of a microscope and the object of interest, $f$, is an image such that $d=2$. We assume that $k$ is finitely smooth, which is equivalent to a polynomial decay of its Fourier coefficients. Futhermore, if we choose $\mathcal{U}$ to be of wavelettype, then the specific structure of the convolution ensures that $\mathcal{W}$ is as in (4) [cf. (29) and (30) below]. Consequently, in this situation we may choose the dictionaries $\mathcal{U}$ and $\mathcal{W}$ such that each $\varphi_{i} \geq 0$ has compact $\operatorname{support} \operatorname{supp}\left(\varphi_{i}\right) \subset\left[\mathbf{t}_{i}-\mathbf{h}_{i}, \mathbf{t}_{i}\right]$. Consequently, if $f \geq 0$, we find

$$
\left\langle f, \varphi_{i}\right\rangle>0 \Rightarrow \exists \mathbf{x} \in\left[\mathbf{t}_{i}-\mathbf{h}_{i}, \mathbf{t}_{i}\right] \quad \text { s.t. } \quad f(\mathbf{x})>0,
$$


that is, there must be a point $\mathbf{x} \in[\mathbf{t}-\mathbf{h}, \mathbf{t}]$ belonging to the support of $f$. Employing this, we can use MISCAT to segment $f$ into active and (most likely) inactive parts, which is of particular interest in many imaging modalities.

With this setup, MISCAT will be used to infer on the location of fluorescent markers in DNA origami imaged by a super-resolution STED microscope [cf. Hell (2007)]. In STED microscopy, the specimen is illuminated by a laser beam along a grid with a diffraction-limited spot centered at the current grid point and the entire specimen is scanned this way, pixel by pixel, leading to observations as in (1) with a convolution $T$ as in (11). The error distribution and the kernel $k$ in (11) are well known experimentally; see the Supplementary Material [Proksch, Werner and Munk (2018)] for a detailed description of the mathematical model.

The investigated specimen consists of DNA origami, which have been designed in a way such that each of the clusters contains up to 24 fluorescent markers, arrayed in two strands of up to 12 having a distance of 71 nanometers (nm) (cf. the sketch in the upper left of Figure 1). As the ground truth is basically known, this serves as a real world phantom. Data were provided by the lab of Stefan Hell of the Department of NanoBiophotonics of the Max Planck Institute for Biophysical Chemistry; cf. Figure 1.

To infer on the positions of the fluorescent markers, we apply MISCAT with a set of scales defined by boxes of size $k_{x} \times k_{y}$ pixels, $k_{x}, k_{y}=4,6, \ldots, 20$. One pixel in the measurements in Figure 1 is of size $10 \mathrm{~nm} \times 10 \mathrm{~nm}$. To highlight our multiscale approach, we also display results of a single scale version of MISCAT [see Remark 2(b) and Section 4.2] using only boxes of size $4 \times 6$ pixels (these are the smallest boxes found by MISCAT), and to highlight the deconvolution effect, we apply a direct multiscale scanning test not designed for deconvolution [i.e., $T=\mathrm{id}$ in the model (1) and $\Phi_{i}=\varphi_{i}$ in (7)] based on indicator functions as

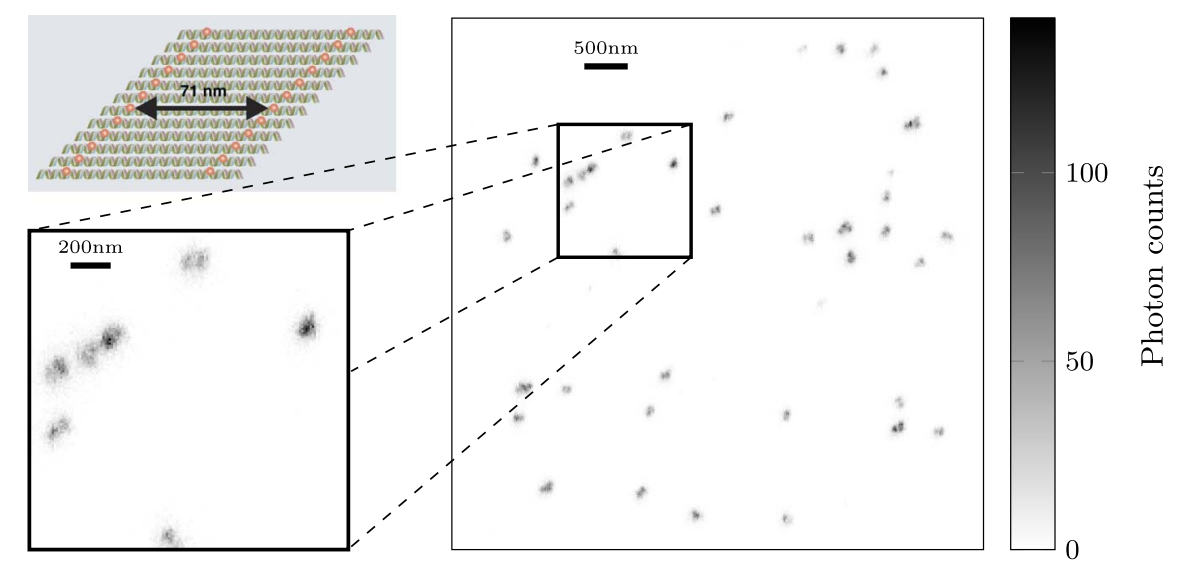

FIG. 1. Experimental data of the DNA origami sample and zoomed region $(150 \times 150$ pixels $)$. The sketch in the upper left shows the structure of the investigated DNA origami sample [red dots represent possible positions for fluorophores; see Ta et al. (2015)]. 


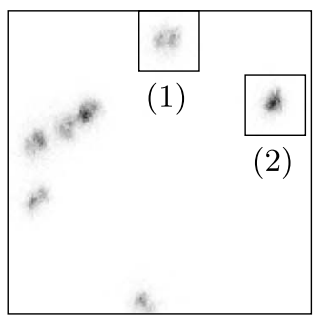

(a) data

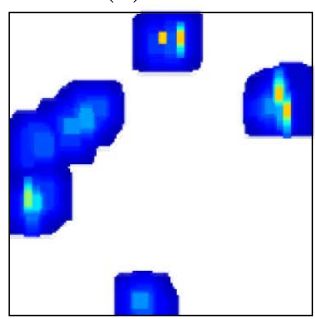

(d) MISCAT

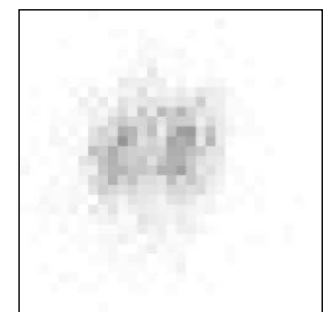

(b) zoomed data, (1)

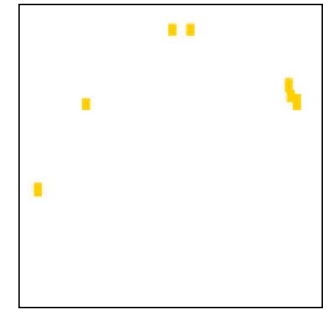

(e) single scale dec. test
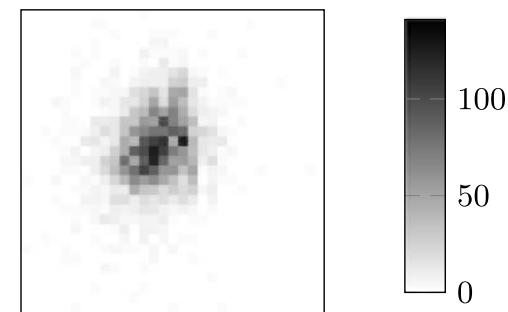

(c) zoomed data, (2)

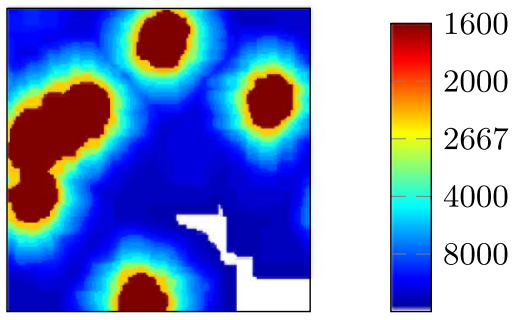

(f) multisc. direct test

FIG. 2. Experimental data and corresponding $90 \%$ significance maps computed by different tests. The color-coding of the significance maps always show the size of smallest significance in $\mathrm{nm}^{2}$, cf. the main text. (a)-(c) data and zoomed regions, (d) MISCAT, (e) a single scale test with deconvolution, (f) a multiscale scanning test without deconvolution. We emphasize that MISCAT performs 1,587,600 tests on the data in (a), and out of those 13,973 local hypotheses are rejected. The FWER control ensures that with (asymptotic) probability at least $90 \%$ among the selected regions there is no wrong detection.

probe functionals using the scale calibration suggested by Dümbgen and Spokoiny (2001); for details, see Section 4.2.

In Figure 2, the zoomed region of Figure 1 is shown together with significance maps for all three tests. The significance map color-codes for each pixel the smallest scale (volume of the box in $\mathrm{nm}^{2}$ ) on which it is significant. In case that a pixel belongs to significant boxes of different scales, only the smallest one is displayed for ease of visualization by the color coding. For instance, in Figure 2(d) MISCAT marked several boxes as significant, and the smallest scale on which significant boxes were found is of size $2400 \mathrm{~nm}^{2}$ (yellow). These results show that MISCAT is able (at least for some of the single DNA origamis) to distinguish both strands. In view of the zoomed data in Figure 2(b) and Figure 2(c), this is quite remarkable as not visible from the data. The latter is due to the fact that the distance between the two strands of $71 \mathrm{~nm}$ is slightly smaller than the full width at half maximum (FWHM, see the Supplementary Material [Proksch, Werner and Munk (2018)] for details) of the convolution kernel $k(\approx 76 \mathrm{~nm})$, and there is a common understanding that objects which are closer to each other than a distance of approximately the FWHM cannot be identified as separate objects. Hence, MISCAT allows to discern objects below the resolution level of the STED microscope. The single scale variant of MISCAT (for explanation see Section 4) in Figure 2(e) has 
clearly more power in detecting small features on this single scale. While the multiscale test detects 4 boxes of $4 \times 6$ pixels, the single scale test detects several more, however, at the price of overseeing many DNA origamis at different scales. Note that the investigated specimen consists only of structures, which are present on a few (known) scales. For illustrative purposes, MISCAT, as employed here, does not use this information, as in general, these scales are not a priori known in living cell imaging. It is also clearly visible in Figure 2(f) that ignoring the deconvolution does not lead to a competitive test: distinguishing between different DNA origamis fails completely, as the support of the DNA-origami has been severely blurred by the STED microscope. We emphasize that the FWER control in (3) with $\alpha=0.1$ implies that with (asymptotic) probability $\geq 90 \%$, each of the 13,973 detections out of 1,587,600 local tests in Figure 2(d) is correct.

\section{General theory.}

2.1. Framework and notation. Recall the general framework introduced in Section 1 and model (1) and that all quantities may depend on the sample size $n$. Throughout this paper, $\left\{T f\left(\mathbf{x}_{\mathbf{j}, n}\right) \mid \mathbf{j} \in I_{n}^{d}\right\}$ is the discretization of the function $T f$ on the grid $\left\{\left(j_{1} / n, \ldots, j_{d} / n\right) \mid 1 \leq j_{k} \leq n, 1 \leq k \leq d\right\}$. This discretization model is a prototype for many inverse problems and in particular matches the application to imaging considered in Section 4 below. For different applications, alternative discretization schemes may be of interest as well but, for the sake of a clearer display, we consider uniform sampling on a complete grid since most of the results presented below do not crucially depend on the specific discretization. We make the following assumption on the dictionaries $\mathcal{U}$ and $\mathcal{W}$ in (2) and (4).

Assumption 1. Let $\mathcal{U}$ as in (2) and $\mathcal{W}$ as in (4).

(a) Dictionary source condition. Let

$$
\varphi_{i} \in \mathcal{R}\left(T^{*}\right), \quad \text { that is, } \varphi_{i}=T^{*} \Phi_{i} .
$$

(b) Growth of the dictionary. For some $\kappa>0$,

$$
|\mathcal{U}|=|\mathcal{W}|=N=O\left(n^{\kappa}\right) .
$$

(c) Scale restrictions. For the smallest and the largest scale in (4), that is, $\mathbf{h}_{\min }=\left(h_{\min }, \ldots, h_{\min }\right)^{T}$ and $\mathbf{h}_{\max }=\left(h_{\max }, \ldots, h_{\max }\right)^{T}$, respectively,

(SR) $\quad h_{\min } \gtrsim n^{-1} \log (n)^{15 / d \vee 3} \log \log (n)^{2} \quad$ and $\quad h_{\max }=o\left(\log (n)^{-2}\right)$.

(d) Average Hölder condition. Suppose that the functions $\Phi_{\mathbf{h}_{i}}$ in (4) are uniformly bounded, supported on $[0,1]^{d}$, vanishing at the boundary and

(AHC)

$$
\int\left|\Phi_{\mathbf{h}_{i}}(\mathbf{t}-\mathbf{z})-\Phi_{\mathbf{h}_{i}}(\mathbf{s}-\mathbf{z})\right|^{2} \mathrm{~d} \mathbf{z} \leq L\|\mathbf{t}-\mathbf{s}\|_{2}^{2 \gamma}\left\|\Phi_{\mathbf{h}_{i}}\right\|_{2}^{2}
$$

for some $\gamma \in(0,1]$ and all $i \in I_{N}$ uniformly as $n$ and hence $N \rightarrow \infty$. 
REMARK 1. (a) Assumption (DSC) is a smoothness condition on the functions of the dictionary $\mathcal{U}$ related to $T$. Instead of posing such an assumption on the dictionary, it is common to pose such an assumption on $f$, that is, the so-called benchmark source condition $f \in \mathcal{R}\left(T^{*}\right)$, which requires the unknown solution $f$ to be at least as smooth as any function in the range of $T^{*}$. For deconvolution problems with real-valued kernel, this means that $f$ is at least as smooth as the kernel itself. In this paper, as we want to reconstruct pairings $\left\langle f, \varphi_{i}\right\rangle$ instead of $f$, we may relax this and pose conditions on the functions $\varphi_{i}$ instead of $f$; see also Burger, Flemming and Hofmann (2013). Note, that if additionally $f$ admits a sparse representation w.r.t. the dictionary $\mathcal{U}$, then (DSC) implies $f \in \mathcal{R}\left(T^{*}\right)$. We emphasize that our approach strongly relies on the condition (DSC); see also Anderssen (1986), Donoho (1995). For a strategy how to estimate a linear functional $\langle f, \varphi\rangle$ for $\varphi \notin R\left(T^{*}\right)$, we refer to Mathé and Pereverzev (2002).

(b) Assumption $(\mathrm{G})$ is rather mild. In particular, it implies that positions and scales $\left(\mathbf{t}_{i}, \mathbf{h}_{i}\right)$ from any grid of polynomial size can be used. In the example of imaging, this is naturally satisfied as the $\mathbf{t}_{i}$ are grid points of the pixel grid and the sizes of the scales $\mathbf{h}_{i}$ are given by rectangular groups of pixels and are hence also only of polynomial order in $n$. Furthermore, to serve as an approximation for a continuous version, the grid can be chosen sufficiently fine and still (G) is satisfied. The constant $\kappa$ only enters into our results via some constants.

As already discussed in the Introduction, the scale restrictions (SR) are also rather mild. The lower bound on $h_{\min }$ is up to a poly-log factor of the same order as the sampling error, and the upper bound on $h_{\max }$ is required to ensure asymptotic unbiasedness of our local test statistics. For some of the results presented below, a slightly stricter bound on $h_{\max }$ will be necessary, and this is emphasized in the corresponding theorems.

(c) Assumption $(\mathrm{AHC})$ is a smoothness condition on the dictionary $\mathcal{W}$. In the case $\gamma<1$, the class of functions satisfying Assumption (AHC) corresponds to the class $H_{2}^{(\gamma, \ldots, \gamma)}$, defined by Nikol'skiĭ [(1951), pages 256-257]; see also Tsybakov (2009), page 13. It holds, for instance, if all $\Phi_{\mathbf{h}_{i}}$ are Hölder-continuous of order $\gamma$. In case $T=\mathrm{id}$, the "classical" scanning function $\Phi_{\mathbf{h}_{i}} \equiv I_{(0,1)}$ ' satisfies condition (AHC) with $\gamma=1 / 2$ and $L=d$. In Section 3, we discuss this condition in more detail and show its validity if $T$ is the Radon transform and if $T$ is a convolution operator (see Section 3.1 and Section 3.2, resp.).

The following assumptions concern the noise $\xi_{\mathbf{j}}, \mathbf{j} \in I_{n}^{d}$ in model (1).

Assumption 2. Let $\xi_{\mathbf{j}}, \mathbf{j} \in I_{n}^{d}$ in (1) be independent and centered random variables. Assume that there exists a function $\sigma \in C^{1}[0,1]^{d}$ such that $\operatorname{Var}\left[\xi_{\mathbf{j}}\right]=$ $\sigma^{2}\left(\mathbf{x}_{\mathbf{j}}\right)$ and

$$
\mathbb{E}\left|\xi_{\mathbf{j}}\right|^{2 J} \leq \frac{1}{2} J ! \mathbb{E} \xi_{\mathbf{j}}^{4} \quad \text { for all } J \geq 2
$$


Assume further that

$$
0<\liminf _{n \rightarrow \infty} \inf _{\mathbf{j} \in I_{n}^{d}} \mathbb{E}\left[\left|\xi_{\mathbf{j}}\right|^{2}\right] \quad \text { and } \quad \limsup _{n \rightarrow \infty} \sup _{\mathbf{j} \in I_{n}^{d}} \mathbb{E}\left[\left|\xi_{\mathbf{j}}\right|^{4}\right]<\infty
$$

Note that (M1) is in fact equivalent to the well-known Cramér condition that the moment generating function exists in a small neighborhood of 0 [cf. Lin (2017), Theorem 1] and is satisfied by many distributions, including Gaussian and Poisson. The latter is most relevant for our subsequent application.

2.2. Asymptotic theory. We are now in the position to provide some general asymptotic properties of MISCAT such as a uniform Gaussian approximation of the test statistic, a.s. boundedness of the simulated quantiles, and weak convergence under further specification of assumptions towards an explicit Gumbel-type distribution. The latter is for ease of presentation only shown when using the full set of possible scales. If MISCAT is restricted to smaller subsets of scales (e.g., resulting from prior information), this may change the limit distribution; see Remark 2 below.

THEOREM 1. Suppose we are given observations from model (1) with random noise satisfying Assumption 2 and dictionaries $\mathcal{U}$ and $\mathcal{W}$ as specified in Assumption 1. Let $h_{\max } \leq n^{-\delta}$ for some (small) $\delta>0$ in (SR) and suppose that the approximation error of $\left\langle\mathbb{E}[Y], \Phi_{i}\right\rangle_{n}:=\frac{1}{n^{d}} \sum_{\mathbf{j} \in I_{n}^{d}} T f\left(\mathbf{x}_{\mathbf{j}}\right) \Phi_{i}\left(\mathbf{x}_{\mathbf{j}}\right)$ is asymptotically negligible, that is,

$$
n^{\frac{d}{2}} \max _{i \in I_{N}} \frac{\left\langle\mathbb{E}[Y], \Phi_{i}\right\rangle_{n}-\left\langle T f, \Phi_{i}\right\rangle}{\left\|\Phi_{i}\right\|_{2}}=o\left(\frac{1}{\log (n)^{2} \log \log (n)^{2}}\right) .
$$

For any constant $K>0$ and $C_{d}=2 d+d / \gamma-1$, consider the calibration values $\omega_{i}=\omega_{i}\left(K, C_{d}\right)$ as in (8).

(a) Then, for a standard Brownian sheet $W$ on $[0,1]^{d}$, it holds

$$
\lim _{n \rightarrow \infty}\left|\mathbb{P}_{0}(\mathcal{S}(Y) \leq q)-\mathbb{P}_{0}(\mathcal{S}(W) \leq q)\right|=0, \quad q \in \mathbb{R},
$$

where $\mathcal{S}(Y)$ and $\mathcal{S}(W)$ are defined in (7) and (10), respectively. Consequently, under $H_{0}, \mathcal{S}(Y)$ and $\mathcal{S}(W)$ converge weakly towards the same limit. Furthermore, the approximating statistic $\mathcal{S}(W)$ is almost surely bounded and does not depend on any unknown quantity.

(b) Instead of (AHC) assume the stronger condition that there exists a function $\Xi$ supported on $[0,1]^{d}$ with $\|\Xi\|_{2}=1$ such that

$$
\max _{i \in I_{N}}\left|\int\left(\frac{\Phi_{\mathbf{h}_{i}}\left(\mathbf{t}_{i}-\mathbf{z}\right)}{\left\|\Phi_{\mathbf{h}_{i}}\right\|_{2}}-\Xi\left(\mathbf{t}_{i}-\mathbf{z}\right)\right) \mathrm{d} W_{\mathbf{z}}\right|=o \mathbb{P}\left(\frac{1}{\sqrt{\log (n)}}\right)
$$


and

$$
\int\left|\Xi\left(D_{\Xi}(\mathbf{t}-\mathbf{z})\right)-\Xi\left(D_{\Xi}(\mathbf{s}-\mathbf{z})\right)\right|^{2} \mathrm{~d} \mathbf{z}=\sum_{j=1}^{d}\left|t_{j}-s_{j}\right|^{2 \gamma}(1+o(1))
$$

with $\gamma \in(0,1]$ and a symmetric, positive definite matrix $D_{\Xi} \in \mathbb{R}^{d \times d}$. Suppose that the set of scales $\mathcal{H}:=\left\{\mathbf{h}_{i} \mid i \in I_{N}\right\}$ is complete, that is, $\mathcal{H}=\left\{h_{\min }, \ldots, h_{\max }\right\}^{d}$, where

$$
\begin{aligned}
& -\log \left(h_{\max }\right)=\delta \log (n)+o(\log (n)), \\
& -\log \left(h_{\min }\right)=\Delta \log (n)+o(\log (n))
\end{aligned}
$$

with $0<\delta<\Delta \leq 1$. If the grids of positions $\mathbf{t}$ and scales $\mathbf{h}$ are furthermore sufficiently fine, that is,

$$
\max _{i \in I_{N}} \min _{j \in I_{N}: \mathbf{t}_{i} \neq \mathbf{t}_{j}}\left\|\mathbf{t}_{i}-\mathbf{t}_{j}\right\|_{\infty}=O\left(n^{-1}\right)
$$

and

$$
\max _{i \in I_{N}} \min _{j \in I_{N} ; h_{i, l} \neq h_{j, l}}\left|\left(h_{j, l}-h_{i, l}\right) / \sqrt{h_{i, l} h_{j, l}}\right| \rightarrow 0 \quad \text { for all } 1 \leq l \leq d
$$

then it holds

$$
\lim _{n \rightarrow \infty} \mathbb{P}_{0}(\mathcal{S}(Y) \leq \lambda)=\exp \left(-\exp (-\lambda) \cdot \frac{H_{2 \gamma} \operatorname{det}\left(D_{\Xi}^{-1}\right) I_{d}(\delta, \Delta)}{\sqrt{2 \pi} K}\right)
$$

with

$$
I_{d}(\delta, \Delta):=\frac{(-1)^{d-1}}{(d-1) !} \sum_{k=0}^{d}(-1)^{k}\left(\begin{array}{l}
d \\
k
\end{array}\right) \log (k \delta+(d-k) \Delta)>0
$$

and Pickands' constant $H_{2 \gamma}$ [cf. Pickands (1969)].

Detailed proofs are deferred to the Supplementary Material [Proksch, Werner and Munk (2018)], and the main ideas are described in Section 6.

REMARK 2. (a) Assumption (13) is a mild assumption on the integral approximation as the required rate is very slow. It is satisfied, in particular, if $T f$ and $\Phi$ in (4) are Hölder-continuous of some order, or if $T f$ is Hölder-continuous and $\Phi$ is an indicator function. Note that due to the ill-posedness of the problem, $T f$ being Hölder-continuous does typically not require $f$ to be continuous.

(b) Although it might seem marginal, a proper choice of the constant $C_{d}$ is crucial for the boundedness of $\mathcal{S}(W)$. The choice $C_{d}=2 d+d / \gamma-1$ used in the formulation of the theorem is adjusted to the case where a dense grid of scales in the sense of (18) is considered. In particular, this includes the case where all scales in Assumption 1 (SR) ranging from $\mathbf{h}_{\min }$ to $\mathbf{h}_{\max }$ are used. If now, for instance, 
$T=\mathrm{id}$ and $\Phi$ in (4) is chosen to be the indicator function of $[0,1]^{d}$, we have $\gamma=1 / 2$, and consequently $C_{d}=4 d-1$, which coincides with the constant of Sharpnack and Arias-Castro (2016) for the Gaussian case.

However, in many situations a less dense grid of scales might be of interest, for example, under prior scale information on the object of interest $f$. Then for the choice $C_{d}=2 d+d / \gamma-1$ the statistic $\mathcal{S}(W)$ is still a.s. bounded from above, but (19) might not be valid anymore. To avoid this, $C_{d}$ has to be adjusted. Suppose in what follows that the grid of positions still satisfies (17). In the least dense regime, when $\mathcal{S}(W)$ behaves as in a single scale scenario, the proper choice is $C_{d}=d / \gamma-1$. Another interesting special case is when only squares in a dense range are considered [this is $\mathbf{h}_{i}=\left(h_{i}, \ldots, h_{i}\right)$ and (18) is satisfied], where one should choose $C_{d}=1+d / \gamma$.

All these choices of $C_{d}$ are specified in more detail in Corollary 1 in Section 5 and follow from our general result in Theorem 7.

(c) As specified in the theorem, $\mathcal{S}(W)$ is bounded for any choice of the constant $K>0$. In fact, $K$ does not affect the asymptotic power of MISCAT as it only determines the location of the limiting distribution. For $\gamma \in\{1 / 2,1\}, H_{2 \gamma}$ can be computed explicitly [see Pickands (1969)], that is, $H_{1}=1$ and $H_{2}=\pi^{-\frac{d}{2}}$. In these cases, the explicit choice $K=\left|\operatorname{det} D_{\Xi}^{-1}\right| I_{d}(\delta, \Delta) H_{2 \gamma} / \sqrt{2 \pi}$ yields standard Gumbel limit distributions. If $\gamma=1$ and if the correlation function $r \Xi$ of the Gaussian field $Z_{\mathbf{t}}=\int \Xi(\mathbf{t}-\mathbf{z}) \mathrm{d} W_{\mathbf{z}}$ is twice differentiable in $\mathbf{0}$, the matrix $D_{\Xi}$ can be computed via $D_{\Xi}^{*} D_{\Xi}=\operatorname{Hess}_{r_{\Xi}}(\mathbf{0})^{-1}$. For $T$ being the Radon transform or a convolution operator, this allows us to give explicit constants $K$ in (27) and (37), respectively, ensuring standard Gumbel limit distributions.

(d) In the situation of Theorem 1(b) under a weaker assumption than (14) and (15), it can be shown that the limiting distribution is stochastically bounded by Gumbel distributions and is hence nondegenerate in the limit. This will be done in Theorem 4 in the situation of deconvolution.

2.3. Statistical inference. In the following, let $q_{1-\alpha}$ denote the $1-\alpha$-quantile of the approximating process $\mathcal{S}(W)$. To compare the local test statistics $\mathcal{S}(Y, i)$ in (7) with $q_{1-\alpha}$, we have assumed so far to know the local variances $\sigma_{i}^{2}=$ $\operatorname{Var}\left[\left\langle Y, \Phi_{i}\right\rangle_{n}\right]$. The next remark shows that they can easily be estimated without changing the limiting distribution of $\mathcal{S}(W)$.

REMARK 3. As mentioned before, the local variances $\sigma_{i}^{2}, i \in I_{N}$, depend on $\operatorname{Var}\left[\xi_{\mathbf{j}}\right]=\sigma^{2}\left(\mathbf{x}_{\mathbf{j}}\right)$ (cf. Assumption 2$), \mathbf{j} \in I_{n}^{d}$, which are typically unknown in applications. Nevertheless, all results remain valid if the $C^{1}$-function $\sigma^{2}$ (see Assumption 2) can be estimated from the data by $\hat{\sigma}^{2}$ such that

$$
\max _{i \in I_{N}}\left|\hat{\sigma}^{2}\left(\mathbf{t}_{i}\right)-\sigma^{2}\left(\mathbf{t}_{i}\right)\right|=o_{\mathbb{P}}\left(\log (n)^{-\frac{1}{2}}\right) .
$$


The local variances $\sigma_{i}^{2}$ can then be estimated by $\hat{\sigma}_{i}^{2}:=\left\langle\hat{\sigma}^{2}, \Phi_{i}^{2}\right\rangle_{n}$. Condition (V) is, for example, satisfied for (suitable) kernel-type estimators or point-wise maximum likelihood estimators as used in Section 4.2.

We conclude by Theorem 1 that $\lim _{n \rightarrow \infty} \mathbb{P}_{0}\left(\mathcal{S}(Y, i) \leq q_{1-\alpha} \forall i \in I_{N}\right) \geq 1-\alpha$, and hence (3) is valid, that is, all rejections are significant findings. Conversely, it can be shown that, with overall confidence of approximately $(1-\alpha) \cdot 100 \%$, all relevant components are found, provided that the signal is sufficiently strong.

LEMMA 1. Suppose we are given observations from model (1) with random noise satisfying Assumption 2 and dictionaries $\mathcal{U}$ and $\mathcal{W}$ as specified in Assumption 1 . Let $\mathcal{I}_{\alpha}$ denote the set of all large components, that is,

$$
\mathcal{I}_{\alpha}:=\left\{i \mid\left\langle\varphi_{i}, f\right\rangle>2\left(\frac{q_{1-\alpha}}{\omega_{i}}+\omega_{i}\right) \sigma_{i}\right\} .
$$

Then, under the assumptions of Theorem 1,

$$
\lim _{n \rightarrow \infty} \mathbb{P}\left(\mathcal{S}(Y, i)>q_{1-\alpha} \text { for all } i \in \mathcal{I}_{\alpha}\right) \geq 1-\alpha .
$$

For general $T$, it is not clear if the detection guarantee in Lemma 1 is optimal in the sense that weaker signals cannot be detected by any procedure. However, in the next subsection we will show that in special situations MISCAT obeys an oracle optimality property.

2.4. Asymptotic optimality. For signals built from block signals, the asymptotic power of MISCAT can be computed explicitly which reveals an oracle optimality property of MISCAT in the following sense. Suppose that $f=$ $\mu_{n, \mathbf{h}_{\star}} I_{\left[\mathbf{t}_{\star}-\mathbf{h}_{\star}, \mathbf{t}_{\star}\right]}$. If one knew the correct scale $\mathbf{h}_{\star}$, one would perform a single-scale test in order to find the location $\mathbf{t}_{\star}$. Hence, in this idealized situation, the "oracle scan statistic" $\mathcal{S}^{\star}(Y)$ given by

$$
\mathcal{S}^{\star}(Y)=\sup _{i \in I_{N}} \omega_{\mathbf{h}_{\star}}\left(K, \frac{d}{\gamma}-1\right)\left(\sigma_{i}^{-1}\left\langle Y, \Phi_{\mathbf{h}_{\star}}\left(\frac{\mathbf{t}_{i}-\cdot}{\mathbf{h}_{\star}}\right)\right\rangle_{n}-\omega_{\mathbf{h}_{\star}}\left(K, \frac{d}{\gamma}-1\right)\right)
$$

would be used. Note the different adjustment of weights due to Remark 2(b). It turns out that MISCAT performs as well in terms of its asymptotic power as the oracle test corresponding to $\mathcal{S}^{\star}(Y)$. Moreover, the following theorem guarantees that signals will be detected asymptotically with probability 1 , if $\mu_{n, \mathbf{h}} \geq$ $\max _{\mathbf{t}} \sigma(\mathbf{t})\left(\sqrt{2 \log \left(1 / \mathbf{h}_{\star}\right)}+\beta_{n}\right) n^{-\frac{d}{2}}\left\|\Phi_{i_{\star}}\right\|_{2}$, where $i_{\star}$ is such that $\left(\mathbf{t}_{i}, \mathbf{h}_{i}\right)=\left(\mathbf{t}_{\star}, \mathbf{h}_{\star}\right)$ and $\beta_{n} \rightarrow \infty$. In this setting, if the errors are i.i.d. standard normal and $T=\mathrm{id}$, the single scale test is minimax optimal if $\left\|\Phi_{i}\right\|_{2}=\sqrt{\mathbf{h}_{i}^{\mathbf{1}}}$, which follows from the arguments in Kou (2017) [see also Arias-Castro, Donoho and Huo (2005), Chan and Walther (2013) for related results]. A rigorous proof for the case $d=2, \Phi=I_{[0,1]^{2}}$ 
and $h_{1}=h_{2}$ can be found in Butucea and Ingster (2013). Thus, also the multiscale procedure MISCAT is minimax optimal in this case. If $T \neq \mathrm{id}$, optimality depends on both dictionaries $\mathcal{W}$ and $\mathcal{U}$ and special care has to be put into the choice of dictionary functions. This is discussed in more detail in Section 3.2.1 below. Under general noise, the following can be said.

THEOREM 2 (Asymptotic power of MISCAT). Suppose we are given observations from model (1) with random noise satisfying Assumption 2 and dictionaries $\mathcal{U}=\left\{\varphi_{i} \mid \varphi_{i}(\mathbf{z})=\varphi\left(\left(\mathbf{t}_{i}-\mathbf{z}\right) / \mathbf{h}_{i}\right), \varphi(\mathbf{z})>0, \mathbf{z} \in(0,1)^{d}\right\}$ and $\mathcal{W}$ as specified in Assumption 1. Suppose (16) with $0<\delta<\Delta \leq 1$ and fix a scale $\mathbf{h}_{\star}=\mathbf{h}_{\star}(n) \in$ $\left[\mathbf{h}_{\min }, \mathbf{h}_{\max }\right]$ and a subset $\mathcal{T}_{\star} \subset I_{N}$ such that $\mathbf{h}_{i}=\mathbf{h}_{\star}$ for all $i \in \mathcal{T}_{\star}$. Now consider the set of functions $f$ with support given by the union of all corresponding boxes which are sufficiently strong, that is,

$$
\mathscr{S}_{\mathcal{T}_{\star}}\left(\mathbf{h}_{\star}, \mu_{n}\right):=\left\{f \mid(13) \text { holds, } \operatorname{supp}(f)=\bigcup_{i \in \mathcal{T}_{\star}} I_{\mathbf{t}_{i}, \mathbf{h}_{\star}}, \frac{\left\langle\varphi_{i}, f\right\rangle}{\left\|\Phi_{i}\right\|_{2}} \geq \frac{\mu_{n}}{n^{d / 2}}, i \in \mathcal{T}_{\star}\right\},
$$

where $I_{\mathbf{t}_{i}, \mathbf{h}_{\star}}:=\left[\mathbf{t}_{i}-\mathbf{h}_{\star}, \mathbf{t}_{i}\right]$. Assume that $\sigma \in C^{1}\left([0,1]^{d}\right)$ and $\mathbf{t}_{\star} \in(0,1)^{d}$ where $\mathbf{t}_{\star} \in \operatorname{argmax}\left\{\sigma(\mathbf{t}) \mid \mathbf{t} \in[0,1]^{d}\right\}$ and let $K>0$.

(a) If $\left\{\mathbf{h}_{i} \mid i \in I_{N}\right\}=\left\{\mathbf{h}_{\star}\right\}$, that is, for each $\mathbf{t}$ we consider scanning windows of (correct) size $\mathbf{h}_{\star}$, then MISCAT with the single-scale-calibration $\omega_{i}(K, d / \gamma-1)$ as in (8) [cf. Remark 2(b)] attains power

$$
\begin{aligned}
\inf _{f \in \mathscr{S}_{\mathcal{T}_{\star}\left(\mathbf{h}_{\star}, \mu_{n}\right)} \mathbb{P}_{f}\left(\mathcal{S}^{\star}(Y)>q_{1-\alpha}\right)} & \inf _{f \in \mathscr{S}_{\left\{\mathbf{t}_{\star}\right\}}\left(\mathbf{h}_{\star}, \mu_{n}\right)} \mathbb{P}_{f}\left(\mathcal{S}^{\star}(Y)>q_{1-\alpha}\right) \\
& =\alpha+(1-\alpha) \cdot \bar{\psi}\left(\sqrt{2 \log \left(\frac{1}{\mathbf{h}_{\star}^{\mathbf{1}}}\right)}-\frac{\mu_{n}}{\sigma\left(\mathbf{t}_{\star}\right)}\right)+o(1) .
\end{aligned}
$$

Here and in the following, $\bar{\psi}(x):=\int_{x}^{\infty}(2 \pi)^{-1 / 2} \exp \left(-y^{2} / 2\right) \mathrm{d} y$ is the tail function of the standard normal distribution.

(b) In general, MISCAT with the multiscale-calibration $\omega_{i}(K, 2 d+d / \gamma-1)$ as in (8) satisfies

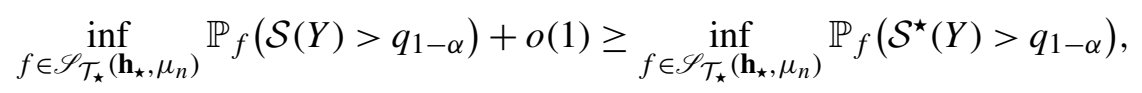

that is, the multiscale procedure performs at least as well as the oracle procedure.

This complements the results from Theorems 4 and 6 in Sharpnack and AriasCastro (2016), where a similar expansion of the power is provided for the case $T=\mathrm{id}$ and $\Phi=I_{[0,1]^{d}}$.

\section{Examples.}


3.1. The d-dimensional Radon transform. Assume one observes a discretized and noisy sample of the Radon transform of $f$,

$$
Y_{\mathbf{k}, l}=T f\left(\boldsymbol{\vartheta}_{\mathbf{k}}, u_{l}\right)+\xi_{\mathbf{k}, l} ; \quad u_{l}=\frac{l-1 / 2}{n}, l=1, \ldots, n
$$

and $\boldsymbol{\vartheta}_{\mathbf{k}} \in \mathbb{S}^{d-1}, \mathbf{k} \in I_{n}^{d-1}$ are design points which are uniformly distributed w.r.t. the angles in a parametrization using polar coordinates, where

$$
T f(u, \vartheta)=\int_{\langle\mathbf{v}, \vartheta\rangle=u} f(\mathbf{v}) \mathrm{d} \mu_{d-1}(\mathbf{v})
$$

denotes Radon transformation [cf. Natterer (1986)], $\mathrm{d} \mu_{d-1}$ denotes the $(d-1)$ dimensional Lebesgue measure on the hyperplane $\{\mathbf{v} \mid\langle\mathbf{v}, \boldsymbol{\vartheta}\rangle=u\}$ and $\xi_{\mathbf{k}, l}$ are i.i.d., $\operatorname{Var}\left[\xi_{(\mathbf{1}, 1)}\right]=\sigma^{2}$. In this case, fix $\widetilde{\varphi}: \mathbb{R}^{+} \rightarrow \mathbb{R}$, set $\varphi(\mathbf{x}):=\widetilde{\varphi}\left(\|x\|_{2}\right)$, $\operatorname{supp}(\tilde{\varphi}) \subset[0,1]$ and define

$$
\mathcal{U}=\left\{\varphi_{i}=h_{i}^{-d / 2} \varphi\left(\frac{\cdot-\mathbf{t}_{i}}{h_{i}}\right) \mid i \in I_{N}\right\}
$$

that is, we consider a dictionary $\mathcal{U}$ of rotationally invariant functions. We now construct the corresponding dictionary $\mathcal{W}$. To this end, we need to fix some more notation. Let $\mathrm{d} \vartheta$ denote the common surface measure on $\mathbf{S}^{d-1}$ such that for measurable $S \subset \mathbb{S}^{d-1}$ we have $|S|=\int_{S} \mathrm{~d} \vartheta$. Let further $\mathcal{F}_{d} f$ denote the $d$-dimensional Fourier transform of $f$, defined by

$\mathcal{F}_{d} f(\boldsymbol{\xi})=\int f(\mathbf{x}) \exp (\mathrm{i}\langle\mathbf{x}, \boldsymbol{\xi}\rangle) \mathrm{d} \mathbf{x}, \quad f(\mathbf{x})=\frac{1}{(2 \pi)^{d}} \int \mathcal{F}_{d} f(\boldsymbol{\xi}) \exp (-\mathrm{i}\langle\boldsymbol{\xi}, \mathbf{x}\rangle) \mathrm{d} \boldsymbol{\xi}$

LEMMA 2. Let $\mathcal{U}$ be as in (23), $\varphi \in \mathcal{R}\left(T^{*}\right)$. Then

$$
\mathcal{W}=\left\{\Phi_{i} \mid \Phi_{i}(u, \boldsymbol{\vartheta})=h_{i}^{-\frac{d}{2}} \Phi\left(\frac{u-\left\langle\boldsymbol{\vartheta}, \mathbf{t}_{i}\right\rangle}{h_{i}}\right)\right\},
$$

where, due to the rotational invariance of $\varphi$, the function $\Phi$, defined by

$$
\Phi(x):=\frac{1}{2(2 \pi)^{d}} \mathcal{F}_{1}\left(\left(\mathcal{F}_{d} \varphi\right)(\cdot \vartheta)|\cdot|^{d-1}\right)(x), \quad x \in \mathbb{R},
$$

is independent of $\vartheta$.

Consequently, the functions $\Phi_{h_{i}}$ as in (4) are in $\mathcal{W}$ as in (24), that is, we have the special structure $\Phi_{h_{i}}=C_{h_{i}} \Phi$, and hence we can define $\Xi:=$ $\Phi_{h_{i}} /\left\|\Phi_{h_{i}}\right\|_{L^{2}\left(\mathbb{R} \times \mathbb{S}^{d-1}\right)}$. It turns out that (AHC) and (15) are satisfied if $\varphi$ is sufficiently smooth. This is made more precise in the following lemma.

LEMMA 3. Let $4 \pi\left\|\mathcal{F}_{1}\left(\left(\mathcal{F}_{d} \varphi\right)(\cdot \boldsymbol{\vartheta})|\cdot|^{d-1}\right)\left(u-\left\langle\mathbf{t}_{i}, \boldsymbol{\vartheta}\right\rangle\right)\right\|_{L^{2}\left(\mathbb{R} \times \mathbb{S}^{d-1}\right)}^{-1}=: C_{\varphi, d}$. If $\varphi \in H^{\frac{d+1}{2}}\left(\mathbb{R}^{d}\right),(15)$ holds with

$$
D_{\Xi}^{-2}:=\operatorname{diag}\left(C_{\varphi, d} \int_{\mathbb{R}^{d}} \omega_{1}^{2}\|\boldsymbol{\omega}\|^{d-1}\left|\left(\mathcal{F}_{d} \varphi\right)(\boldsymbol{\omega})\right|^{2} \mathrm{~d} \boldsymbol{\omega}\right)
$$


In general, the dictionary functions $\Phi$ may be of unbounded support. In this case, the results from Theorem 1(b) remain valid if we exclude a small boundary region from our analysis. Here, we only consider positions $\mathbf{t}_{i} \in[\mathbf{0}, \mathbf{1}-\boldsymbol{\rho}]$, where $\boldsymbol{\rho}=(\rho, \ldots, \rho)^{T}, \rho>0$ and we obtain the following extreme value theorem for MISCAT in the case of the Radon transform.

THEOREM 3 (MISCAT for the Radon transform). Suppose that we have access to observations following model (22). Let $\mathbf{t}_{i} \in[\mathbf{0}, \mathbf{1}-\boldsymbol{\rho}]$, where $\boldsymbol{\rho}=$ $(\rho, \ldots, \rho)^{T}, \rho>0$. Assume also that the approximation error of $\left\langle\mathbb{E}[Y], \Phi_{h_{i}}\right\rangle_{n}$ is asymptotically negligible, that is, (13) holds and $\varphi \in H^{\frac{d+1}{2}}\left(\mathbb{R}^{d}\right)$, such that the integral in (26) is finite. If furthermore (16) holds true with $0<\delta<\Delta \leq 1$ and the grids of positions $\mathbf{t}$ and scales $h$ are sufficiently fine, that is, satisfy (17) and (18) and if the calibration

$$
\omega(K, 1+d) \quad \text { with } K=(1-\rho)^{d}(2 \pi)^{-\frac{d+1}{2}} \operatorname{det}\left(D_{\Xi}^{-2}\right)^{\frac{1}{2}} \log (\Delta / \delta)
$$

is used [see (8) and Remark 2(b)], where $D_{\Xi}^{-2}$ is defined in (26), then one has $\lim _{n \rightarrow \infty} \mathbb{P}_{0}[\mathcal{S}(Y) \leq \lambda]=e^{-e^{-\lambda}}$. Furthermore, the statements of Lemma 1 and Theorem 2 also hold.

3.2. Deconvolution. We discuss now in detail the case of deconvolution, that is, (1) specializes to

$$
Y_{\mathbf{j}}=(k * f)\left(\mathbf{x}_{\mathbf{j}}\right)+\xi_{\mathbf{j}}, \quad \mathbf{j} \in\{1, \ldots, n\}^{d},
$$

where the function $k$ is a convolution kernel and the operation " $*$ " denotes convolution as defined in (11). In our subsequent data example, $k$ corresponds to the point-spread function (PSF) of a microscope [see, e.g., Bertero et al. (2009), Aspelmeier, Egner and Munk (2015), Hohage and Werner (2016)].

Assume that there exist positive constants $\underline{c}, \bar{C}$ and $a$ such that

$$
\underline{c}\left(1+\|\boldsymbol{\xi}\|_{2}^{2}\right)^{-a} \leq\left|\mathcal{F}_{d} k(\boldsymbol{\xi})\right| \leq \bar{C}\left(1+\|\boldsymbol{\xi}\|_{2}^{2}\right)^{-a} .
$$

Assumption (D1) is a standard assumption characterizing mildly ill-posed deconvolution problems [see, e.g., Fan (1991), Meister (2009)]. For any fixed function $\varphi,\|\varphi\|_{2}>0$, generating a dictionary

$$
\mathcal{U}=\left\{\varphi_{i} \mid \varphi_{i}(\mathbf{z})=\varphi\left(\frac{\mathbf{t}_{i}-\mathbf{z}}{\mathbf{h}_{i}}\right), i \in I_{N}\right\},
$$

the corresponding dictionary $\mathcal{W}$ inherits the required wavelet-type structure:

$$
\mathcal{W}=\left\{\Phi_{i} \mid \Phi_{i}(\mathbf{z})=\Phi_{\mathbf{h}_{i}}\left(\frac{\mathbf{t}_{i}-\mathbf{z}}{\mathbf{h}_{i}}\right), \Phi_{\mathbf{h}_{i}}:=\mathcal{F}_{d}^{-1}\left(\frac{\mathcal{F}_{d} \varphi}{\overline{\mathcal{F}_{d} k\left(\cdot / \mathbf{h}_{i}\right)}}\right), i \in I_{N}\right\},
$$

and the results from the previous section transfer to deconvolution as follows. 
THEOREM 4 (MISCAT for deconvolution). Suppose model (28) with convolution kernel $k$ satisfying Assumption (D1) and $\xi_{\mathbf{j}}$ satisfying Assumption 2. Let $\mathbf{t}_{i} \in\left[\boldsymbol{\rho}+\mathbf{h}_{i}, \mathbf{1}-\boldsymbol{\rho}\right]$, where $\boldsymbol{\rho}=(\rho, \ldots, \rho)^{T}, \rho>0$. Consider the dictionary $\mathcal{W}$, given by (30) such that Assumption 1 is satisfied and, in addition, $\varphi$ belongs to a Sobolev space $H^{2 a+\gamma \vee 1 / 2}\left(\mathbb{R}^{d}\right)$. Assume further that the approximation error of $\left\langle\mathbb{E}[Y], \Phi_{i}\right\rangle_{n}$ is asymptotically negligible, that is, (13) holds.

(a) The results of Theorem 1(a) carry over to this general setting.

(b) Furthermore, let the grids of positions $\mathbf{t}$ and scales $\mathbf{h}$ sufficiently fine, that is, satisfy (17) and (18). Then there exist positive constants $\underline{D}_{\gamma}$ and $\bar{D}_{\gamma}$ such that for any fixed $\lambda \in \mathbb{R}$

$$
e^{-\underline{D}_{\gamma} e^{-\lambda}} \leq \lim _{n \rightarrow \infty} \mathbb{P}_{0}[\mathcal{S}(Y) \leq \lambda] \leq e^{-\bar{D}_{\gamma} e^{-\lambda}}
$$

Hence, under $H_{0}, \mathcal{S}(Y)$ is asymptotically nondegenerate.

(c) In the situation of (b), let $\mathbf{h}_{i}=\left(h_{i}, \ldots, h_{i}\right)$ for all $i \in I_{N}$ and assume that (16) holds true with $0<\delta<\Delta \leq 1$. If the stronger condition (14) holds, then with the calibration $w(K, 1+d)$ we obtain

$$
\lim _{n \rightarrow \infty} \mathbb{P}_{0}(\mathcal{S}(Y) \leq \lambda)=\exp \left(-\exp (-\lambda) \cdot \frac{H_{2 \gamma} \operatorname{det}\left(D_{\Xi}^{-1}\right) \log (\Delta / \delta)}{\sqrt{2 \pi} K}\right) .
$$

REMARK 4. (a) In Theorem 4, we need to exclude a small boundary region of the observations from the analysis since, in general, the functions $\Phi_{\mathbf{h}_{i}}$ in $\mathcal{W}$ might be of unbounded support. Then the results of Theorem 1 transfer to this setting.

(b) The results from Theorem 4 (c) require assumption (14) which basically means that the convolution kernel $k$ should decay exactly like a polynomial if $\|\boldsymbol{\xi}\|_{2} \rightarrow \infty$ in contrast to the weaker assumption (D1) which only requires upper and lower polynomial bounds and can hence only ensure upper and lower Gumbel bounds. In Section 4, we provide a specific example for which both (D1) and (14) are satisfied.

3.2.1. Optimal detection in deconvolution. In this section, we discuss and specify the results from Sections 2.3 and 2.4 for deconvolution. The results given in Lemma 1 also hold in the general deconvolution setting. The following lemma contains a related result in the situation of (32) concerning the support inference about the signal $f$ itself.

LEMMA 4. Given observations from model (28) with random noise satisfying Assumption 2 and $k$ as in (32) and given a nonnegative function $\varphi \in \mathcal{R}\left(T^{*}\right)$, define the dictionary $\mathcal{W}$ as in (30). Suppose that the signal $f$ is nonnegative as well. Let further $\mathcal{I}_{\alpha}(f)$ denote the set

$$
\mathcal{I}_{\alpha}(f):=\left\{i|f|_{\operatorname{supp}\left(\varphi_{i}\right)}>2 q_{i, 1-\alpha}\left\|\sigma \Phi_{i}\right\|_{2} /\left(h_{i, 1} h_{i, 2} n^{d / 2}\right)\right\} .
$$


Then, under the assumptions of Theorem 1 ,

$$
\lim _{n \rightarrow \infty} \mathbb{P}\left(\left\langle\Phi_{i}, Y\right\rangle_{n}>q_{i, 1-\alpha}\left\|\sigma \Phi_{i}\right\|_{2} / n \text { for all } i \in \mathcal{I}_{\alpha}(f)\right) \geq 1-\alpha .
$$

The result above immediately shows that the choice of $\varphi$ in (29) has a high influence on the detection properties of the corresponding test via the variances $\left\|\sigma \Phi_{i}\right\|_{2}^{2}$. Extending an argument from Schmidt-Hieber, Munk and Dümbgen (2013) for $d=1$ to general $d$, we can provide a mother wavelet $\varphi$ which minimizes the asymptotic variance of the test statistic over all tensor-type probe functions. It only depends on the polynomial order of decay of the convolution kernel in Fourier space ( $\hat{=}$ degree of ill-posedness) and is (for $d=2$ ) given by

$$
\varphi(x, y)=x^{\beta_{1}+1}(1-x)^{\beta_{1}+1} y^{\beta_{2}+1}(1-y)^{\beta_{2}+1} \mathbf{1}_{(0,1)}(x) \mathbf{1}_{(0,1)}(y),
$$

where the two parameters $\beta_{1}, \beta_{2} \in \mathbb{N}$ equal the polynomial order of decay of the convolution kernel in $x$ and $y$ direction. This choice will be considered in the following.

The previous lemma implies the consistency of the testing procedure for the signal itself, that is, testing $f=0$ versus $f>0$, if the minimal scale satisfies $h_{\min } \gtrsim(\log (n) / n)^{1 /(4 a+1)}$. Moreover, in the situation of Theorem 5(c) the optimality results of Section 2.4 carry over to the deconvolution setting. For a comparison, consider the rate of estimation of the $2 a$ th derivative of a Hölder $\beta$ function w.r.t. $L^{\infty}$ risk in $d=1$. We restrict to this case as otherwise the deconvolution is no longer equivalent to estimating derivatives; cf. (33). This is possible with minimax rate $(\log n / n)^{\beta /(2 \beta+4 a+1)}$, which is attained for $h \sim(\log n / n)^{1 /(2 \beta+4 a+1)}$ [see, e.g., Johnstone et al. (2004)], that is, such a function can be distinguished from 0 by means of estimation on a box $[t-h, t]$ as long as it is asymptotically larger than $h^{\beta}$. Posing the same question to MISCAT, the above result shows that for $\left.f\right|_{[t-h, t]} \sim h^{\beta}$ and $h \sim(\log n / n)^{1 /(2 \beta+4 a+1)}$ it recognizes $[t-h, t]$ as active with (asymptotic) probability $\geq 1-\alpha$. Consequently, any support points found by estimation will also be found by MISCAT.

4. Simulations and real data applications. In this section, we investigate the finite sample properties of the proposed multiscale test. To this end, we apply MISCAT in a 2-dimensional mildly ill-posed deconvolution problem. In Section 4.2, we then analyze experimental STED data to locate single DNA origami in a sample.

Specifying the setting described in Section 3.2 to this situation, the data is given by (28). The convolution kernel $k$ is chosen from the parametric family $\left\{k_{a, b} \mid\right.$ $a \in \mathbb{N}, b>0\}$ defined in Fourier space via

$$
\left(\mathcal{F}_{2} k_{a, b}\right)(\boldsymbol{\xi})=\left(1+b^{2}\|\boldsymbol{\xi}\|_{2}^{2}\right)^{-a}, \quad \boldsymbol{\xi} \in \mathbb{R}^{2} .
$$

Model (32) is a 2-dimensional generalization of the one-dimensional family of auto-convolutions of a scaled version of the density of the Laplace distribution 
with itself with radially symmetric PSF. For any convolution kernel $k_{a, b}$ Assumption (D1) is obviously satisfied and we obtain

$$
\Phi_{\mathbf{h}_{i}}=\sum_{j=0}^{a} \sum_{k=0}^{j}\left(\begin{array}{l}
a \\
j
\end{array}\right)\left(\begin{array}{l}
j \\
k
\end{array}\right)\left(\frac{b}{h_{i, 1}}\right)^{2 k}\left(\frac{b}{h_{i, 2}}\right)^{2(j-k)} \partial^{(2 k, 2(j-k))} \varphi .
$$

Alternatively, the functions $\Phi_{\mathbf{h}_{i}}$ can be computed by means of the Fourier transform as in (30). However, (33) shows that a compactly supported function $\varphi$ results in a dictionary $\mathcal{W}$ which consists of compactly supported functions as well. Consequently, the results from Theorem 4 can be obtained even without excluding a small boundary region, and furthermore a Gumbel limit theorem can be obtained as follows. Let

$$
\Xi=\frac{\widetilde{\Xi}}{\|\widetilde{\Xi}\|_{2}} \quad \text { where } \widetilde{\Xi}=b^{2 a} \sum_{k=0}^{a}\left(\begin{array}{l}
a \\
k
\end{array}\right) \partial^{2 k, 2(a-k)} \varphi,
$$

and consider the case $\mathbf{h}_{i}=\left(h_{i}, h_{i}\right)$ for all $i \in I_{N}$. Then

$$
\left\|\Phi_{\mathbf{h}_{i}}\right\|_{2}=\left(\frac{1}{h_{i}}\right)^{2 a}\left\|\boldsymbol{\Xi}+h_{i}^{2} \Xi_{n, i}\right\|_{2} \quad \text { and } \quad \frac{\Phi_{\mathbf{h}_{i}}}{\| \Phi_{\mathbf{h}_{i} \|_{2}}}=\frac{\Xi+h_{i}^{2} \Xi_{n, i}}{\left\|\Xi+h_{i}^{2} \Xi_{n, i}\right\|_{2}},
$$

where

$$
\Xi_{n, i}:=\sum_{j=0}^{a-1} h_{i}^{2(a-1-j)} \sum_{k=0}^{j}\left(\begin{array}{l}
j \\
k
\end{array}\right)\left(\begin{array}{l}
a \\
j
\end{array}\right) \partial^{2 k, 2(j-k)} \varphi .
$$

In this setting, it is easy to verify that condition (14) holds.

THEOREM 5 (MISCAT for our application). Suppose that we have access to observations following model (28) with convolution kernel $k_{a, b}$ satisfying Assumption (32), $d=2$ and random noise satisfying Assumption 2. Assume that the dictionary is given by (30) with dictionary functions $\Phi_{\mathbf{h}_{i}}$ defined in (33) such that Assumption 1 is satisfied, and that (13) holds.

(a) The results of Theorem 1(a) carry over to this particular convolution setting.

(b) If $\varphi \in H^{2 a+\gamma \wedge 1 / 2}\left(\mathbb{R}^{2}\right)$ and if the grids of positions $\mathbf{t}$ and scales $\mathbf{h}$ are sufficiently fine, that is, satisfy (17) and (18), then the results of Theorem 4(b) carry over to this particular convolution setting.

(c) Suppose furthermore that $\mathbf{h}_{i}=\left(h_{i}, h_{i}\right)$ for all $i \in I_{N}$, that (16) holds true with $0<\delta<\Delta \leq 1$ and that the grids of positions $\mathbf{t}$ and scales $\mathbf{h}$ are sufficiently fine, that is, satisfy (17) and (18). If in addition, $\varphi$ is $(2 a+1)$-times differentiable in $L^{2}\left(\mathbb{R}^{d}\right)$, let $\varphi_{\boldsymbol{\alpha}}=\sum_{k=0}^{a}\left(\begin{array}{l}a \\ k\end{array}\right) \partial^{2 k+\alpha_{1}, 2(a-k)+\alpha_{2}} \varphi, \boldsymbol{\alpha} \in\{0,1\}^{2},|\boldsymbol{\alpha}|=1$. Then, for $\omega(K, 1+d)$ with

$$
K=b^{4 a} \log (\Delta / \delta)(2 \pi)^{-\frac{3}{2}}\|\widetilde{\Xi}\|_{2}^{-1} \sqrt{\left\|\varphi_{0,1}\right\|_{2}^{2}\left\|\varphi_{1,0}\right\|_{2}^{2}-\left\langle\varphi_{0,1} \varphi_{1,0}\right\rangle}
$$

[see (8) and Remark 2(b)], we obtain $\lim _{n \rightarrow \infty} \mathbb{P}_{0}[\mathcal{S}(Y) \leq \lambda]=e^{-e^{-\lambda}}$. 
4.1. 2-dimensional support inference. In the Supplementary Material [Proksch, Werner and Munk (2018)], we present detailed simulations to infer on the support of a testfunction of size $512 \times 512$, that is, $n=512$ with a kernel $k_{a, b}$ as in (32). This setting is close to our subsequent data example. We apply MISCAT using 196 different scales defined by boxes consisting of $k_{x} \times k_{y}$ pixels, $k_{x}, k_{y}=4,6, \ldots, 30$. Concerning the positions $\mathbf{t}$ we use again all possible upper left points of boxes fitting in the image, which results in 48,219,136 local tests in total. To implement MISCAT, we first fix $\varphi$ as in (31), and then compute the 196 functions $\Phi_{\mathbf{h}_{i}}$ as in (30) by using the Fourier convolution theorem, that is, $\Phi_{\mathbf{h}_{i}}=\mathcal{F}_{d}^{-1}\left(\frac{\mathcal{F}_{d} \varphi}{\mathcal{F}_{d} k_{a, b}\left(\cdot / \mathbf{h}_{i}\right)}\right)$ as in (30). This can be done explicitly exploiting the structure of $k_{a, b}$, and is efficiently implemented using FFT, which results in $\mathcal{O}\left(196 \cdot 512^{2} \log \left(512^{2}\right)\right)$ flops. Similarly, all local statistics $\left\langle Y, \Phi_{i, n}\right\rangle_{n}$ with fixed scale $\mathbf{h} \equiv \mathbf{h}_{i}$ can also be computed by two FFTs using the Fourier convolution theorem. Consequently, MISCAT for deconvolution problems can be performed in general in $\mathcal{O}$ (\#scales · \#pixels $\log$ (\#pixels)), and in the setting here the evaluation of the roughly 50 million local test statistics takes less than one minute on a standard laptop. Finally, to perform MISCAT, the (asymptotic) quantiles of the approximating Gaussian test statistic $\mathcal{S}(W)$ can be pre-computed, which corresponds to many evaluations of the maximum in (10) and is costly.

Let us now briefly conclude the findings in the Supplementary Material [Proksch, Werner and Munk (2018)]. First of all, our simulations suggest a nondegenerated behavior of the distribution of the penalized maximum statistic.

Concerning support inference we observe that MISCAT with correctly specified degree of ill-posedness [this is $\beta_{1}=\beta_{2}=2 a$ in (31) with $a$ as in (32)] is able to detect large objects even in a large noise regime, and for sufficiently small amount of noise it is even able to separate objects which have a distance of 9 pixels, which is even less than the FWHM (see the Supplementary Material [Proksch, Werner and Munk (2018)]). We furthermore find that misspecification of ill-posedness does not provide false detections, but loss in detection power, where underspecification of the ill-posedness [ $\beta_{1}=\beta_{2}=1$ in (31)] has less severe effects to MISCAT than overspecification $\left[\beta_{1}=\beta_{2}=10\right.$ in (31)]. This can also be seen from Figure 3 where a synthetic testfunction, simulated data from a homogeneous Gaussian model with noise level $\sigma=0.05$ and significance maps of the three corresponding tests (correctly specified, overspecified and underspecified ill-posedness) is shown. The significance map color-codes for each pixel the smallest scale on which it was significant; for details, see the Supplementary Material [Proksch, Werner and Munk (2018)].

Finally, we also investigate robustness of MISCAT to the noise distribution in the Supplementary Material [Proksch, Werner and Munk (2018)]. We investigate empirical levels under the student's t distribution $t(v)$, which has $v-1$ moments, and hence does not satisfy (M1) for any $v$. Nevertheless, for sufficiently large parameter $v$ we still obtain an empirical level close to our theoretical value $\alpha$. 


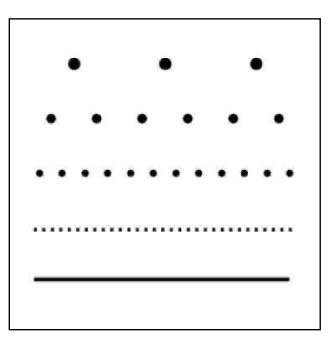

(a) test function

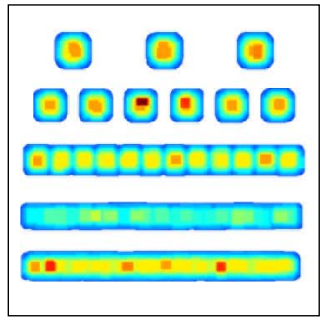

(c) $\beta_{1}=\beta_{2}=4$
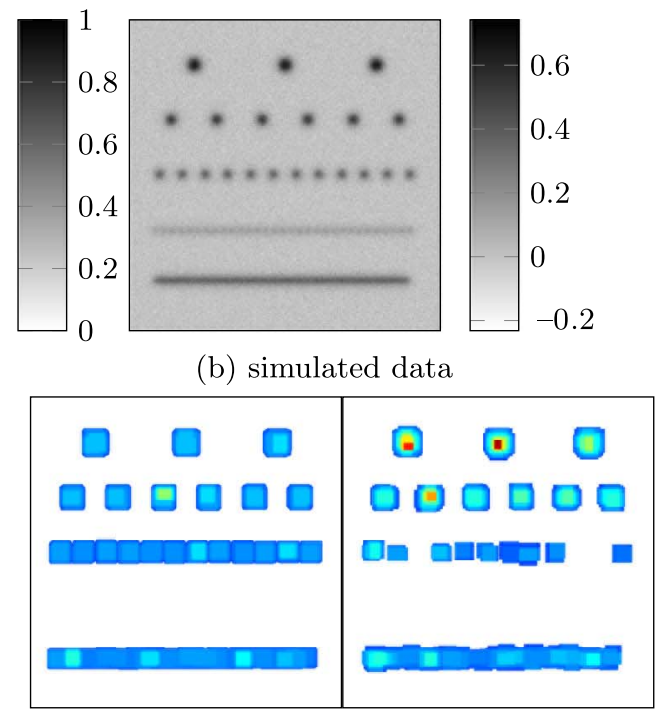

(d) $\beta_{1}=\beta_{2}=10$
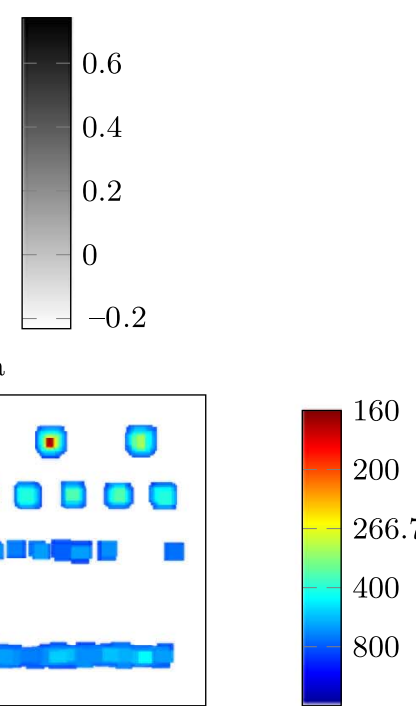

(e) $\beta_{1}=\beta_{2}=1$

FIG. 3. (a) Synthetic test function, (b) simulated data from a homogeneous Gaussian model with noise level $\sigma=0.05$, (c) 90\%-significance map of MISCAT with correctly specified ill-posedness $\left[\beta_{1}=\beta_{2}=4\right.$ in (31)], (d) 90\%-significance map of MISCAT with overspecified ill-posedness [ $\beta_{1}=\beta_{2}=10$ in (31)], (e) 90\%-significance map of MISCAT with underspecified ill-posedness $\left[\beta_{1}=\beta_{2}=1\right.$ in (31)]. The color-coding shows the smallest scale (in pixels) on which the corresponding pixel was significant.

Furthermore, we investigate a model mimicking data coming from CCD sensors, which consists of Poissonian observations which are additionally corrupted by additive Gaussian noise. This model satisfies (M1), and our simulations suggest that MISCAT keeps its level quite stable over a large range of parameters. Only in the situation of a low Poisson intensity, the heavier tail behavior of the Poisson distribution dominates and the empirical level deteriorates.

4.2. Locating fluorescent markers in STED super-resolution microscopy. Based on the results from Section 4.1, we are now able to rigorously treat the real world application from Section 1.2 from 2-dimensional STED (stimulated emission depletion) super-resolution microscopy [Hell and Wichmann (1994), Klar and Hell (1999), Hell (2007)]. A brief overview over the experimental setup is already given in the Introduction, and for a detailed mathematical model we refer to the Supplementary Material [Proksch, Werner and Munk (2018)], where we argue there that our measurements are described reasonably by

$$
Y_{\mathbf{j}} \stackrel{\text { independent }}{\sim} \operatorname{Bin}\left(t,\left(k_{2,0.016} * f\right)\left(\mathbf{x}_{\mathbf{j}}\right)\right), \quad \mathbf{j} \in\{1, \ldots, 600\}^{2} .
$$

Here, $\operatorname{Bin}(t, p)$ denotes the Binomial distribution with parameters $t \in \mathbb{N}$ and $p \in$ $[0,1]$, observations are obtained on the grid $\left\{\mathbf{x}_{\mathbf{j}} \mid \mathbf{j} \in\{1, \ldots, 600\}^{2}\right\}$ and $f(\mathbf{x})$ is the 
probability that a photon emitted at grid point $\mathbf{x}$ is recorded at the detector in a single excitation pulse. The kernel $k_{2,0.016}$ is as in (32), and in actual experiments $t$ is roughly $10^{3}$.

With this kernel, we design a test using the optimal probe function $\varphi$ in (31) (i.e., $\beta_{1}=\beta_{2}=4$ ) and a set of scales defined by boxes of size $k_{x} \times k_{y}$ pixels, $k_{x}, k_{y}=4,6, \ldots, 20$, resulting in $28,100,601$ local tests. The variances $\sigma_{i}^{2}$ in (7) used in the test statistic are estimated from the data point-wise using a maximum likelihood estimator. Furthermore, we ease the problem by neglecting all boxes in which no photons where observed, that is, we drop all pairs $\left(\mathbf{t}_{i}, \mathbf{h}_{i}\right)$ such that $Y_{\mathbf{j}}=0$ for all $\mathbf{x}_{\mathbf{j}} \in\left[\mathbf{t}_{i}-\mathbf{h}_{i}, \mathbf{t}_{i}\right]$. Even though this choice is data dependent, and hence random, the uniformity over all pairs $\left(\mathbf{t}_{i}, \mathbf{h}_{i}\right)$ of our confidence statements ensures that those stay valid.

With this test, we analyze the data shown in Figure 1; cf. Section 1.2 for details. In total, MISCAT marks 94,141 out of 28,100,601 boxes as significant. For a comparison, we also use two different tests, namely an analog of MISCAT using only one single scale of size $4 \times 6$ pixels [these are the smallest boxes found by MISCAT, see Remark 2(b)], and the multiscale scanning test ignoring the deconvolution ( $T=\mathrm{id})$, boiling down to the test statistic of Dümbgen and Spokoiny (2001):

$$
\max _{i} \frac{\sqrt{\log \left(3 / \mathbf{h}_{i}^{\mathbf{1}}\right)}}{\log \left(\log \left(3 / \mathbf{h}_{i}^{\mathbf{1}}\right)\right)}\left[\frac{1}{\sqrt{\mathbf{h}_{i}^{\mathbf{1}}}} \sum_{\mathbf{x}_{\mathbf{j}} \in\left[\mathbf{t}_{i}-\mathbf{h}_{i}, \mathbf{t}_{i}\right]} Y_{\mathbf{j}}-\sqrt{2 \log \left(3 / \mathbf{h}_{i}^{\mathbf{1}}\right)}\right]
$$

For all tests, we again use empirical quantiles computed in $10^{4}$ runs of the test statistics applied to Gaussian white noise.

The full result is depicted in Figure 4. As mentioned in the Introduction, MISCAT is able (at least for some of the single DNA origamis) to infer on position and rotation as indicated in the first panel in Figure 4. Remarkably, this information is not visible by eye; cf. Figure 2 .

5. Multiscale extreme value theory. In this section, we state the results that are the core of the proofs of our theorems from the previous sections. The following theorem guarantees that the Gaussian approximation $\mathcal{S}(W)$ is asymptotically bounded from above almost surely.

Let $Z_{\mathbf{t}, \mathbf{h}}:=1 / \sqrt{\mathbf{h}^{\mathbf{1}}} \int \Xi\left(\frac{\mathbf{t}-\mathbf{z}}{\mathbf{h}}\right) \mathrm{d} W_{\mathbf{z}}$.

THEOREM 6 (MISCAT: a.s. boundedness). Let $\Xi$ be a normed function, that is, $\|\Xi\|_{2}=1$, supported on $[0,1]^{d}$ such that (AHC) holds. Let $(\mathbf{t}, \mathbf{h}) \in \mathcal{H} \times \mathcal{T}_{\mathbf{h}} \subset$ $\left[\mathbf{h}_{\min }, \mathbf{h}_{\max }\right] \times[\mathbf{h}, \mathbf{1}]$, where $h_{\max } \leq n^{-\delta}$. There exists a function $F$ which is independent of $n$, such that $\lim _{\lambda \rightarrow \infty} F(\lambda)=0$ and for $\lambda>0$ :

$$
\mathbb{P}\left(\sup _{\mathbf{h} \in \mathcal{H}} \sup _{\mathbf{t} \in \mathcal{T}_{\mathbf{h}}} \omega_{\mathbf{h}}\left(Z_{\mathbf{t}, \mathbf{h}}-\omega_{\mathbf{h}}\right)>\lambda\right) \leq F(\lambda) .
$$



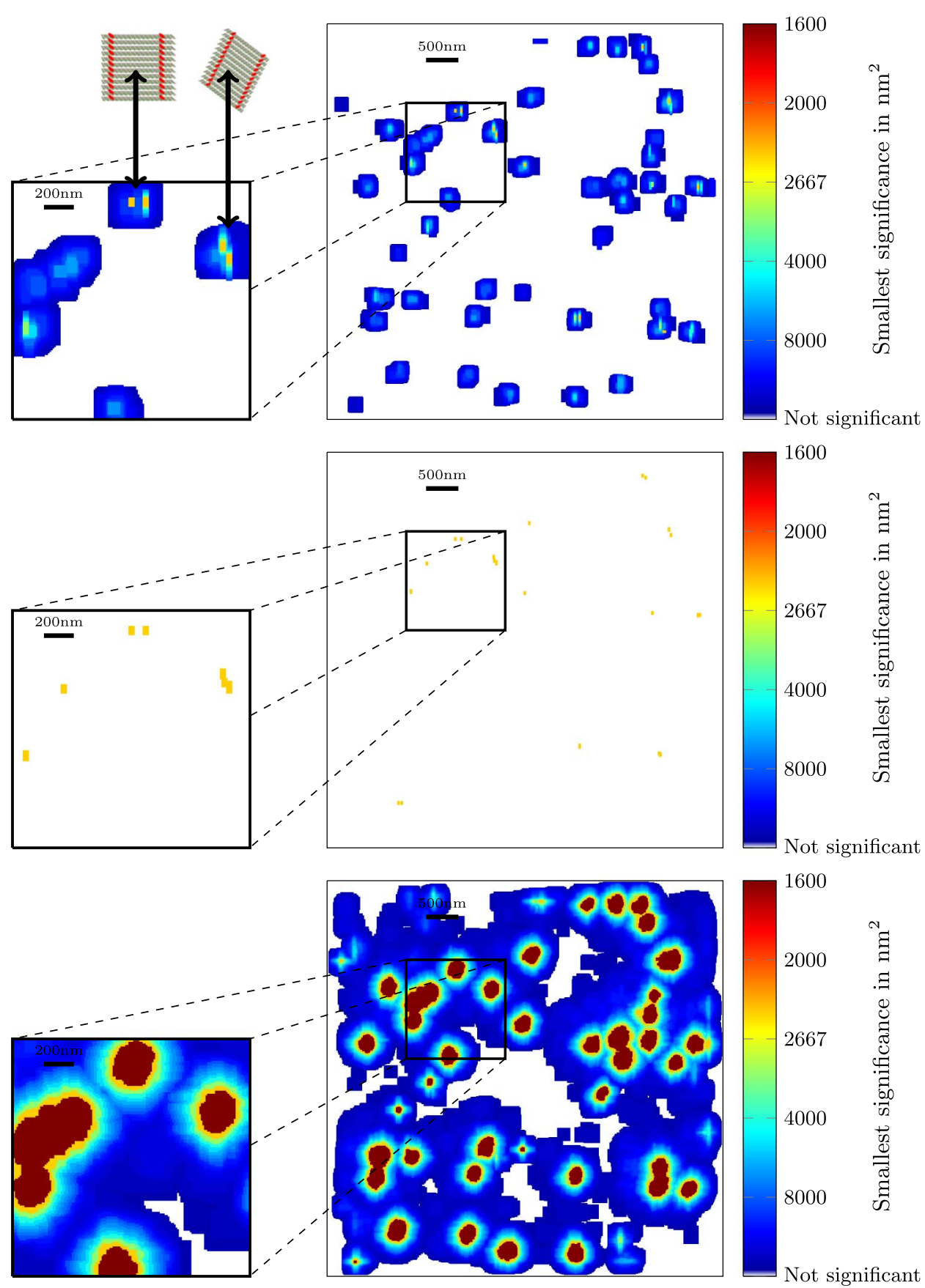

FIG. 4. $90 \%$ significance maps and excerpts for different tests computed from the data in Figure 1. From top to bottom: MISCAT, a single scale test with deconvolution, and the standard multiscale test without deconvolution. 
This implies in particular that $\mathcal{S}(W)$ is almost surely bounded. Furthermore, there exists a positive constant $\underline{D}_{\gamma}$ such that for any fixed $\lambda \in \mathbb{R}$,

$$
e^{-\underline{D}_{\gamma} e^{-\lambda}} \leq \lim _{n \rightarrow \infty} \mathbb{P}\left(\sup _{\mathbf{h} \in \mathcal{H}} \sup _{\mathbf{t} \in \mathcal{T}_{\mathbf{h}}} \omega_{\mathbf{h}}\left(Z_{\mathbf{t}, \mathbf{h}}-\omega_{\mathbf{h}}\right) \leq \lambda\right)
$$

The following theorem yields a weak limit for multiscale statistics of the type $\mathcal{S}(W)$.

THEOREM 7 (A general multiscale Gumbel limit theorem). Let $\Xi$ be a normed function, that is, $\|\Xi\|_{2}=1$, supported on $[0,1]^{d}$ such that (15) holds. Let $K>0$ be a fixed, positive constant. If furthermore (16) holds true with $0<\delta<\Delta \leq 1$,

$$
\lim _{n \rightarrow \infty} \mathbb{P}\left(\sup _{\mathbf{h} \in\left[h_{\min }, h_{\max }\right]^{d}} \sup _{\mathbf{t} \in[\mathbf{h}, \mathbf{1}]} \omega_{\mathbf{h}}\left(Z_{\mathbf{t}, \mathbf{h}}-\omega_{\mathbf{h}}\right) \leq \lambda\right)=e^{-e^{-\lambda} \cdot \frac{H_{2 \gamma}\left|\operatorname{det} D_{\Xi}^{-1}\right| I_{d}(\delta, \Delta)}{\sqrt{2 \pi} K},},
$$

where $\omega_{\mathbf{h}}$ and $I_{d}(\delta, \Delta)$ are defined as in (8) and (20), respectively.

Corollary 1 below follows immediately from the proofs of the previous Theorems. Two special cases are discussed in Remark 2.

COROLlary 1. Suppose that the assumptions of Theorem 6 hold. Assume that $\mathbf{h}_{i} \in \mathcal{H}_{1} \times \cdots \times \mathcal{H}_{d}$, where possibly $\mathcal{H}_{i} \neq \mathcal{H}_{j}$ for $i \neq j$. Let for $\mathcal{P}:=$ $\left\{\left\lfloor\log \left(1 / h_{\max }\right)\right\rfloor-2,\left\lfloor\log \left(1 / h_{\max }\right)\right\rfloor-1, \ldots,\left\lceil\log \left(1 / h_{\min }\right)\right\rceil\right\}$, and $j \in\{1, \ldots, d\}$

$$
\mathcal{P}_{j}:=\left\{p \in \mathcal{P} \mid \exists h_{i, j} \in \mathcal{H}_{j}: h_{i, j} \in\left[e^{-(p+1)}, e^{-p}\right)\right\} .
$$

Choose the constant $C_{d}$ in (8) such that there exist positive constants $\underline{d}$ and $\bar{D}$ such that

$$
\underline{d} \leq \log (n)^{-\frac{C_{d}-d / \gamma+1}{2}}\left|\mathcal{P}_{1} \times \cdots \times \mathcal{P}_{d}\right| \leq \bar{D}
$$

(a) The results of Theorem 6 remain valid.

(b) If, in addition, the grid of positions $\mathbf{t}$ is sufficiently fine, that is, (17) holds and for each $j$, the sets $\mathcal{P}_{j}=\mathcal{P}_{j, n}$ are increasing sets with respect to $n \in \mathbb{N}$, that is, $\left|\mathcal{P}_{j, n}\right| \leq\left|\mathcal{P}_{j, n+1}\right|$ and $\sum_{p_{j, n} \in \mathcal{P}_{j, n}} p_{j, n}$ is increasing, there exists a constant $C_{\mathcal{P}}>0$ such that

$$
\lim _{n \rightarrow \infty} \mathbb{P}\left(\sup _{\mathbf{h} \in \mathcal{H}_{1} \times \cdots \times \mathcal{H}_{d}} \sup _{\mathbf{t} \in \mathcal{T}_{\mathbf{h}}} \omega_{\mathbf{h}}\left(Z_{\mathbf{t}, \mathbf{h}}-\omega_{\mathbf{h}}\right) \leq \lambda\right)=e^{-e^{-\lambda} \cdot \frac{H_{2 \gamma}\left|\operatorname{det} D_{\Xi}^{-1}\right| C_{\mathcal{P}}}{\sqrt{2 \pi} K}} .
$$


6. Proofs. This section contains only the key ideas and main steps of the proof of our key result, Theorem 7. A complete version of this proof can be found in the Supplementary Material [Proksch, Werner and Munk (2018)].

PROOF OF THEOREM 7. Recall that with a slight abuse of notation, we denote $\mathbf{h}^{\alpha}=h_{1}^{\alpha_{1}} \cdot \ldots \cdot h_{d}^{\alpha_{d}}, \mathbf{h}_{\mathbf{p}}=\left(h_{1}, \ldots, h_{d}\right)^{T}, \mathbf{1} / \mathbf{h}_{\mathbf{p}}=\left(1 / h_{1}, \ldots, 1 / h_{d}\right)^{T}$ and inequalities between vectors or multi-indices are meant component-wise.

Step I: Proof for scales on a dyadic grid. We show later in Step II.3 that the supremum over $\left[h_{\min }, h_{\max }\right]$ and the supremum over $\left[h_{\min } / \log (n)^{\frac{1}{\gamma}} \log \log (n)\right.$, $\left.h_{\text {max }}\right]$ are asymptotically equivalent and consider first the supremum over the slightly enlarged set. Define a dyadic grid $\mathcal{H}_{\text {dyad }} \subset\left[h_{\min } / \log (n)^{\frac{1}{\gamma}} \log \log (n)\right.$, $\left.h_{\max }\right]$ as follows:

$$
\mathcal{H}_{\text {dyad }}:=\left\{2^{-p} \mid p \in \mathcal{P}\right\}, \quad \mathcal{P}=\left\{\left\lceil b_{\gamma}\left(h_{\max }\right)\right\rceil, \ldots,\left\lfloor b_{\gamma}\left(h_{\min }\right)\right\rfloor\right\},
$$

where $b_{\gamma}(h):=\log \left(\frac{\log (n)^{\frac{1}{\gamma}} \log \log (n)}{h}\right) / \log (2)$. Here and below, $\log$ denotes the natural logarithm. Define $p_{\min }:=\min \mathcal{P}$ and $p_{\max }:=\max \mathcal{P}$. Let

$$
\begin{aligned}
\max _{\mathbf{h} \in \mathcal{H}_{\text {dyad }}^{d}} \sup _{\mathbf{t} \in[\mathbf{h}, \mathbf{1}]} \omega_{\mathbf{h}}\left(\frac{1}{\sqrt{\mathbf{h}^{\mathbf{1}}}} \int \Xi\left(\frac{\mathbf{t}-\mathbf{z}}{\mathbf{h}}\right) \mathrm{d} W_{\mathbf{z}}-\omega_{\mathbf{h}}\right) \\
\stackrel{\mathcal{D}}{=} \max _{\mathbf{h} \in \mathcal{H}_{\text {dyad }}^{d}} \sup _{\mathbf{t} \in[\mathbf{1}, \mathbf{1} / \mathbf{h}]} \omega_{\mathbf{h}}\left(\int \Xi(\mathbf{t}-\mathbf{z}) \mathrm{d} W_{\mathbf{z}}-\omega_{\mathbf{h}}\right)=: M_{n},
\end{aligned}
$$

by stationarity of $Z_{\mathbf{t}, \mathbf{h}}$ for fixed $\mathbf{h}$. We now consider the term $M_{n}$.

Step I.1: Partition of the parameter set. The form of $M_{n}$ in (38) reveals a redundancy pattern that we will exploit later on. Observe that the suprema with respect to $\mathbf{t}$ of the rescaled version $M_{n}$ are taken over subsets of the rectangle $\left[\mathbf{1}, \mathbf{1} / \mathbf{h}_{\min }\right]$. For smaller scales, the supremum with respect to $\mathbf{t}$ is taken over larger sets. Obviously, for $\mathbf{p} \in \mathcal{P}^{d}$,

$$
\left[\mathbf{1} / \mathbf{h}_{\mathbf{p}}, \mathbf{1} / \mathbf{h}_{\mathbf{p}+\mathbf{1}}\right] \subset\left[\mathbf{1}, \mathbf{1} / \mathbf{h}_{\mathbf{s}}\right] \quad \forall \mathbf{s}>\mathbf{p}+\mathbf{1} .
$$

In order to exploit this fact, we partition the parameter set $\left[\mathbf{1}, \mathbf{1} / \mathbf{h}_{\min }\right]$ into suitable blocks, that is, into blocks $B_{\mathbf{p}+\mathbf{1}, \mathbf{q}+\mathbf{1}}$ that are approximately equal to $\left[\mathbf{1} / \mathbf{h}_{\mathbf{p}}, \mathbf{1} / \mathbf{h}_{\mathbf{p}+\mathbf{1}}\right]$ in order to split the suprema with respect to $\mathbf{t}$ into suitable subsuprema. To achieve that those sub-suprema are independent, we separate the blocks by small bands of width 1 . This ensures independence, since $\operatorname{supp}(\Xi) \subset$ $[0,1]^{d}$. The bands only yield a contribution which is asymptotically negligible, which we will show in Step I.3 below.

To be precise, we define subsets of $\left[\mathbf{1}, \mathbf{1} / \mathbf{h}_{\mathrm{min}}\right]$ as follows:

$$
B_{\mathbf{p}}:=\left[\frac{\mathbf{1}}{\mathbf{h}_{\mathbf{p}-1}}, \frac{\mathbf{1}}{\mathbf{h}_{\mathbf{p}}}-\mathbf{1}\right] \text { and } R_{\mathbf{p}}=\left[\frac{\mathbf{1}}{\mathbf{h}_{\mathbf{p}-1}}, \frac{\mathbf{1}}{\mathbf{h}_{\mathbf{p}}}\right] \backslash B_{\mathbf{p}}
$$


where $\mathbf{h}_{\mathbf{p}_{\min }-1}:=\mathbf{1}$. The large blocks $B_{\mathbf{p}}$ yield the main contributions. The sets $R_{\mathbf{p}}$ are asymptotically negligible (see Step I.3 below). Define further for $\mathbf{q} \in \mathcal{P}^{d}$

$$
\mathcal{B}_{\mathbf{q}}:=\bigcup_{\mathbf{p} \in \mathcal{P}^{d}, \mathbf{p} \leq \mathbf{q}} B_{\mathbf{p}} \quad \text { and } \quad M_{\mathcal{B}}:=\max _{\mathbf{p} \in \mathcal{P}^{d}} \omega_{\mathbf{h}_{\mathbf{p}}}\left(M_{\mathcal{B}_{\mathbf{p}}}-\omega_{\mathbf{h}_{\mathbf{p}}}\right) \text {. }
$$

Write

$$
\begin{aligned}
M_{\mathcal{B}} & =\max _{\mathbf{q} \in \mathcal{P}^{d}} \max _{\mathbf{p} \leq \mathbf{q}} \sup _{\mathbf{t} \in B_{\mathbf{p}}} \omega_{\mathbf{h}_{\mathbf{q}}}\left(\int \Xi(\mathbf{t}-\mathbf{z}) \mathrm{d} W_{\mathbf{z}}-\omega_{\mathbf{h}_{\mathbf{q}}}\right) \\
& =\max _{\mathbf{p} \in \mathcal{P}^{d}} \max _{\mathbf{q} \geq \mathbf{p}} \sup _{\mathbf{t} \in B_{\mathbf{p}}} \omega_{\mathbf{h}_{\mathbf{q}}}\left(\int \Xi(\mathbf{t}-\mathbf{z}) \mathrm{d} W_{\mathbf{z}}-\omega_{\mathbf{h}_{\mathbf{q}}}\right) .
\end{aligned}
$$

Fix $\lambda \in \mathbb{R}$. Since the blocks $B_{\mathbf{p}}$ are constructed such that the sub-maxima over different blocks are independent, we find

$$
\begin{aligned}
\mathbb{P}\left(M_{\mathcal{B}} \leq \lambda\right) & =\prod_{\mathbf{p} \in \mathcal{P}^{d}} \mathbb{P}\left(\max _{\mathbf{p} \leq \mathbf{q}} \sup _{\mathbf{t} \in B_{\mathbf{p}}} \omega_{\mathbf{h}_{\mathbf{q}}}\left(\int \Xi(\mathbf{t}-\mathbf{z}) \mathrm{d} W_{\mathbf{z}}-\omega_{\mathbf{h}_{\mathbf{q}}}\right) \leq \lambda\right) \\
& =\prod_{\mathbf{p} \in \mathcal{P}^{d}} \mathbb{P}\left(\sup _{\mathbf{t} \in B_{\mathbf{p}}} \int \Xi(\mathbf{t}-\mathbf{z}) \mathrm{d} W_{\mathbf{z}} \leq \Lambda_{\text {min, }}\right),
\end{aligned}
$$

where $\Lambda_{\min , \mathbf{p}}:=\min _{\mathbf{p} \leq \mathbf{q}}\left(\frac{\lambda}{\omega_{\mathbf{h}_{\mathbf{q}}}}+\omega_{\mathbf{h}_{\mathbf{q}}}\right)$. Now we have broken the proof down into $|\mathcal{P}|^{d}$ "one-scale" extreme value problems and use results for those. Let $\operatorname{Leb}\left(B_{\mathbf{p}}\right)$ denote the Lebesgue-measure of $B_{\mathbf{p}}$ and let $\Lambda_{\mathbf{p}}$ denote

$$
\Lambda_{\mathbf{p}}:=\lambda / \omega_{\mathbf{h}_{\mathbf{p}}}+\omega_{\mathbf{h}_{\mathbf{p}}}
$$

For any fixed $\lambda \in \mathbb{R}$, we have that $\Lambda_{\min , \mathbf{p}}=\Lambda_{\mathbf{p}}$, for sufficiently large $n$. Thus,

$$
\mathbb{P}\left(M_{\mathcal{B}} \leq \lambda\right)=\prod_{\mathbf{p} \in \mathcal{P}^{d}}\left(1-\mathbb{P}\left(\sup _{\mathbf{t} \in B_{\mathbf{p}}} \int \boldsymbol{\Xi}(\mathbf{t}-\mathbf{z}) \mathrm{d} W_{\mathbf{z}}>\Lambda_{\mathbf{p}}\right)\right) .
$$

Step I.2: Derivation of the weak limit on the dyadic grid. Next, we estimate

$$
P_{n, \mathbf{p}}(\lambda):=\mathbb{P}\left(\sup _{\mathbf{t} \in B_{\mathbf{p}}} \int \mathbf{\Xi}(\mathbf{t}-\mathbf{z}) \mathrm{d} W_{\mathbf{z}} \leq \Lambda_{\mathbf{p}}\right)
$$

using Theorem 7.2 in Piterbarg (1996). In the Supplementary Material [Proksch, Werner and Munk (2018)], we give the explicit calculations which show that the following holds:

$$
P_{n}(\lambda)=\exp \left(-e^{-\lambda} \frac{H_{2 \gamma}\left|\operatorname{det} D_{\Xi}^{-1}\right|}{K \sqrt{2 \pi}} \sum_{\mathbf{p} \in \mathcal{P}^{d}}\left(\log \left(\frac{K}{\mathbf{h}_{\mathbf{p}}^{\mathbf{1}}}\right)\right)^{-d}\right)(1+o(1))
$$


for some $\delta_{e}>0$. Notice that

$$
\sum_{\mathbf{p} \in \mathcal{P}^{d}}\left(\log \left(\frac{K}{\mathbf{h}_{\mathbf{p}}^{\mathbf{1}}}\right)\right)^{-d} \sim \int_{[\delta \log (n), \Delta \log (n)]^{d}}\left(\frac{1}{\log (K)+z_{1}+\cdots+z_{d}}\right)^{d} \mathrm{~d} \mathbf{z}=: I_{n, d} .
$$

By induction with respect to $d \in \mathbb{N}$, we show in the Supplementary Material that

$$
\frac{(d-1) !}{(-1)^{d}} I_{n, d}=\log \left(\prod_{j \text { even }}(k \delta+(d-k) \Delta)^{\left(\begin{array}{c}
d \\
j
\end{array}\right)}\right)-\log \left(\prod_{j \text { odd }}(k \delta+(d-k) \Delta)^{\left(\begin{array}{l}
d \\
j
\end{array}\right)}\right) \text {. }
$$

Hence, the statement of the theorem holds true for scales on the dyadic grid.

Step I.3: Negligibility of the remainder terms. In the Supplementary Material, we first show that, asymptotically, the slight enlargement of the domain of the scales from the beginning of Step I does not have an impact. Then, we show that the contribution of the separating regions, $\mathcal{R}_{\mathbf{p}}, \mathbf{p} \in \mathcal{P}^{d}$ are asymptotically negligible.

Step II: Show that the dyadic grid is sufficiently dense. We now show

$$
\Delta_{\gamma, n}=\left|\max _{\mathbf{h} \in \mathcal{H}_{\text {dyad }}^{d}} \sup _{\mathbf{t} \in \mathcal{T}} \omega_{\mathbf{h}}\left(Z_{\mathbf{t}, \mathbf{h}}-\omega_{\mathbf{h}}\right)-\sup _{\mathbf{h} \in\left[h_{\min }, h_{\max }\right]^{d}} \sup _{\mathbf{t} \in \mathcal{T}} \omega_{\mathbf{h}}\left(Z_{\mathbf{t}, \mathbf{h}}-\omega_{\mathbf{h}}\right)\right|=o_{\mathbb{P}}(1) .
$$

Let $\varepsilon>0$.

$$
\mathbb{P}\left(\Delta_{n, \gamma}>\varepsilon\right) \leq \mathbb{P}\left(\max _{\mathbf{p} \in \mathcal{P}^{d}}\left|\omega_{\mathbf{h}_{\mathbf{p}}}\left(\sup _{\mathbf{t} \in \mathcal{T}}\left|Z_{\mathbf{t}, \mathbf{h}_{\mathbf{p}}}-Z_{\mathbf{t}, \mathbf{h}}\right|+\max _{\mathbf{h} \in\left[\mathbf{h}_{\mathbf{p}}, \mathbf{h}_{\mathbf{p}+1}\right]}\left|\omega_{\mathbf{h}}-\omega_{\mathbf{h}_{\mathbf{p}}}\right|\right)\right|>\varepsilon\right) .
$$

Step II.1: Fineness of the dyadic grid I. Let $h \in\left[h_{\min }, h_{\max }\right]$. Set $p=$ $\left\lfloor\log \left(\log (n)^{\frac{1}{\gamma}} \log \log (n) / h\right)\right\rfloor$ and assign the element $h_{\text {dyad }}$ of the dyadic grid to $h$ :

$$
h_{\text {dyad }}=\operatorname{argmin}\left\{|g-h| \mid g \in\left\{2^{-p}, \ldots, 2^{-p_{\min }}\right\}\right\} .
$$

We show in the Supplementary Material that $\left|\omega_{\mathbf{h}}-\omega_{\mathbf{h}_{\text {dyad }}}\right|=o(1 / \sqrt{\log (n)})$.

Step II.2: Estimation of the covering numbers. We show in detail in the Supplementary Material that there exists a constant $C_{\text {cov }}$, depending only on the dimension $d$ and the function $\Xi$ via the constants $L_{\Xi}$ and $\gamma$ from condition (AHC) such that for $\varepsilon \in(0, d)$,

$$
\mathcal{N}(\mathcal{T} \times \mathcal{H}, \rho, \varepsilon) \leq C_{\operatorname{cov}}\left(\frac{1}{\varepsilon}\right)^{\frac{2 d}{\gamma}}\left(\frac{1}{h_{\min }}-\frac{1}{h_{\max }}\right)^{d},
$$

where $\rho^{2}((\mathbf{t}, \mathbf{h}),(\mathbf{s}, \mathbf{l}))=\mathbb{E}\left|Z_{\mathbf{t}, \mathbf{h}}-Z_{\mathbf{s}, \mathbf{l}}\right|^{2}$ and $\mathcal{N}(\mathcal{T} \times \mathcal{H}, \rho, \varepsilon)$ denotes the covering numbers of $\mathcal{T} \times \mathcal{H}$ with respect to $\rho$.

Step II.3: Fineness of the dyadic grid II. By an application of Dudley's theorem, using the estimates from Step II.2, we show in the Supplementary Material that

$$
\max _{\mathbf{p} \in \mathcal{P}^{d}} \omega_{\mathbf{h}_{\mathbf{p}}}\left|\sup _{\mathbf{t} \in \mathcal{T}} Z_{\mathbf{t}, \mathbf{h}_{\mathbf{p}}}-\sup _{\mathbf{h} \in\left[\mathbf{h}_{\mathbf{p}}, \mathbf{h}_{\mathbf{p}+\mathbf{1}}\right]} \sup _{\mathbf{t} \in \mathcal{T}} Z_{\mathbf{t}, \mathbf{h}}\right|=o(1) .
$$

Hence, the supremum over the dyadic grid and the supremum over the full range $\left[h_{\text {min }}, h_{\text {max }}\right]^{d}$ have the same limit. 
Acknowledgments. We thank Haisen Ta and Jan Keller from the Department of NanoBiophotonics, Max Planck Institute for Biophysical Chemistry, for providing the experimental data and expertise. We also thank Cristina Butucea for helpful comments and several anonymous referees and the editors for a number of insightful questions and constructive suggestions which helped us to improve the quality of the paper substantially.

\section{SUPPLEMENTARY MATERIAL}

Supplement to "Multiscale scanning in inverse problems" (DOI: 10.1214/ 17-AOS1669SUPP; .pdf). This supplementary material contains an explanation of the full width at half maximum (FWHM), a detailed mathematical model for super-resolution STED microscopy, a detailed simulation study for 2-dimensional support inference and detailed proofs of all theoretical results provided in the main document.

\section{REFERENCES}

Abramovich, F. and Silverman, B. W. (1998). Wavelet decomposition approaches to statistical inverse problems. Biometrika 85 115-129. MR1627226

Albani, V., Elbau, P., De Hoop, M. V. and Scherzer, O. (2016). Optimal convergence rates results for linear inverse problems in Hilbert spaces. Numer. Funct. Anal. Optim. 37 521-540.

ANDERSSEN, R. S. (1986). The linear functional strategy for improperly posed problems. In Inverse Problems (Oberwolfach, 1986). Internat. Schriftenreihe Numer. Math. 77 11-30. Birkhäuser, Basel. MR0902807

Arias-Castro, E., Donoho, D. L. and Huo, X. (2005). Near-optimal detection of geometric objects by fast multiscale methods. IEEE Trans. Inform. Theory 51 2402-2425. MR2246369

Aspelmeier, T., Egner, A. and Munk, A. (2015). Modern statistical challenges in highresolution fluorescence microscopy. Annu. Rev. Stat. Appl. 2 163-202.

Bertero, M., BocCACCI, P., DesiderÀ, G. and Vicidomini, G. (2009). Image deblurring with Poisson data: From cells to galaxies. Inverse Probl. 25025004.

Bissantz, N., Hohage, T., MunK, A. and RuYmgaART, F. (2007). Convergence rates of general regularization methods for statistical inverse problems and applications. SIAM J. Numer. Anal. 45 2610-2636.

Bissantz, N., Claeskens, G., Holzmann, H. and Munk, A. (2009). Testing for lack of fit in inverse regression-With applications to biophotonic imaging. J. R. Stat. Soc. Ser. B. Stat. Methodol. 71 25-48. MR2655522

Burger, M., Flemming, J. and Hofmann, B. (2013). Convergence rates in regularization if the sparsity assumption fails. Inverse Probl. 29025013.

ButUCEA, C. (2007). Goodness-of-fit testing and quadratic functional estimation from indirect observations. Ann. Statist. 35 1907-1930.

Butucea, C. and Comte, F. (2009). Adaptive estimation of linear functionals in the convolution model and applications. Bernoulli 15 69-98.

Butucea, C. and IngSter, Y. I. (2013). Detection of a sparse submatrix of a high-dimensional noisy matrix. Bernoulli 19 2652-2688.

CASTILlO, I. and NiCKL, R. (2014). On the Bernstein-von Mises phenomenon for nonparametric Bayes procedures. Ann. Statist. 42 1941-1969. MR3262473

Cavalier, L. and Golubev, Y. (2006). Risk hull method and regularization by projections of ill-posed inverse problems. Ann. Statist. 34 1653-1677. MR2283712 
CAVALIER, L. and Tsybakov, A. (2002). Sharp adaptation for inverse problems with random noise. Probab. Theory Related Fields 123 323-354.

Cavalier, L., Golubev, Y., LePski, O. and Tsybakov, A. (2003). Block thresholding and sharp adaptive estimation in severely ill-posed inverse problems. Teor. Veroyatn. Primen. 48 534-556.

Chan, H. P. and Walther, G. (2013). Detection with the scan and the average likelihood ratio. Statist. Sinica 23 409-428.

Chernousova, E. and Golubev, Y. (2014). Spectral cut-off regularizations for ill-posed linear models. Math. Methods Statist. 23 116-131.

Chernozhukov, V., Chetverikov, D. and Kato, K. (2014). Gaussian approximation of suprema of empirical processes. Ann. Statist. 42 1564-1597. MR3262461

Cohen, A., Hoffmann, M. and Reiss, M. (2004). Adaptive wavelet Galerkin methods for linear inverse problems. SIAM J. Numer. Anal. 42 1479-1501.

DedeCKER, J., MERLEVÈdE, F. and Rio, E. (2014). Strong approximation of the empirical distribution function for absolutely regular sequences in $\mathbb{R}^{d}$. Electron. J. Probab. 19 1-56.

Dickhaus, T. (2014). Simultaneous Statistical Inference: With Applications in the Life Sciences. Springer, Heidelberg.

Donoho, D. L. (1995). Nonlinear solution of linear inverse problems by wavelet-vaguelette decomposition. Appl. Comput. Harmon. Anal. 2 101-126.

DümbGen, L. and Spokoiny, V. (2001). Multiscale testing of qualitative hypotheses. Ann. Statist. 29 124-152.

DümbGen, L. and Walther, G. (2008). Multiscale inference about a density. Ann. Statist. 36 1758-1785.

Eckle, K., Bissantz, N. and Dette, H. (2017). Multiscale inference for multivariate deconvolution. Electron. J. Stat. 11 4179-4219. MR3716498

Eckle, K., Bissantz, N., Dette, H., Proksch, K. and Einecke, S. (2018). Multiscale inference for a multivariate density with applications to X-ray astronomy. Ann. Inst. Statist. Math. To appear. DOI:10.1007/s10463-017-0605-1.

FAN, J. (1991). Asymptotic normality for deconvolution kernel density estimators. Sankhyā, Ser. A 53 97-110.

FriedenberG, D. A. and Genovese, C. R. (2013). Straight to the source: Detecting aggregate objects in astronomical images with proper error control. J. Amer. Statist. Assoc. 108 456-468.

Genovese, C. R., Perone-Pacifico, M., Verdinelli, I. and Wasserman, L. (2012). The geometry of nonparametric filament estimation. J. Amer. Statist. Assoc. 107 788-799.

Goldenshluger, A. (1999). On pointwise adaptive nonparametric deconvolution. Bernoulli 5 907-925.

HELL, S. (2007). Far-field optical nanoscopy. Science 316 1153-1158.

HeLl, S. W. and WichmanN, J. (1994). Breaking the diffraction resolution limit by stimulated emission: Stimulated-emission-depletion fluorescence microscopy. Opt. Lett. 19 780-782.

Hohage, T. and Werner, F. (2016). Inverse problems with Poisson data: Statistical regularization theory, applications and algorithms. Inverse Probl. 32093001.

Holzmann, H., BissantZ, N. and MUNK, A. (2007). Density testing in a contaminated sample. J. Multivariate Anal. 98 57-75. MR2292917

INGSTER, Y. I. (1993). Asymptotically minimax hypothesis testing for nonparametric alternatives. I-III. Math. Methods Statist. 2 85-114, 171-189, 249-268.

Ingster, Y., LAUREnT, B. and MARTEAU, C. (2014). Signal detection for inverse problems in a multidimensional framework. Math. Methods Statist. 23 279-305.

IngSter, Y. I., SAPATinAs, T. and Suslina, I. A. (2012). Minimax signal detection in ill-posed inverse problems. Ann. Statist. 40 1524-1549. MR3015034

Johnstone, I. M. and PAUL, D. (2014). Adaptation in some linear inverse problems. Stat 3 187199. 
Johnstone, I. M. and Silverman, B. W. (1991). Discretization effects in statistical inverse problems. J. Complexity 7 1-34.

Johnstone, I. M., Kerkyacharian, G., PiCARD, D. and Raimondo, M. (2004). Wavelet deconvolution in a periodic setting. J. R. Stat. Soc. Ser. B. Stat. Methodol. 66 547-573.

Kabluchko, Z. (2011). Extremes of the standardized Gaussian noise. Stochastic Process. Appl. 121 515-533. MR2763094

Kazantsev, I., Lemahieu, I., Salov, G. and Denys, R. (2002). Statistical detection of defects in radiographic images in nondestructive testing. Signal Process. 82 791-801.

Kerkyacharian, G., Kyriazis, G., Le Pennec, E., Petrushev, P. and Picard, D. (2010). Inversion of noisy Radon transform by SVD based needlets. Appl. Comput. Harmon. Anal. 28 $24-45$.

KLAR, T. A. and HELL, S. W. (1999). Subdiffraction resolution in far-field fluorescence microscopy. Opt. Lett. 24 954-956.

KNAPIK, B. T., VAN DER VAART, A. W. and VAN ZANTEN, J. H. (2011). Bayesian inverse problems with Gaussian priors. Ann. Statist. 39 2626-2657. MR2906881

Komlós, J., MAJOR, P. and TusnÁDY, G. (1975). An approximation of partial sums of independent RV's and the sample DF. I. Z. Wahrsch. Verw. Gebiete 32 111-131.

KoU, J. (2017). Identifying the support of rectangular signals in Gaussian noise. Preprint. Available at arXiv: 1703.06226

Laurent, B., Loubes, J.-M. and Marteau, C. (2011). Testing inverse problems: A direct or an indirect problem? J. Statist. Plann. Inference 141 1849-1861.

Laurent, B., Loubes, J.-M. and Marteau, C. (2012). Non asymptotic minimax rates of testing in signal detection with heterogeneous variances. Electron. J. Stat. 6 91-122.

Li, H., Munk, A., Sieling, H. and Walther, G. (2016). The essential histogram. Preprint. Available at arXiv:1612.07216.

LIN, G. D. (2017). Recent developments on the moment problem. Preprint. Available at arXiv:1703:01027.

MAIR, B. A. and RUYMgAarT, F. H. (1996). Statistical inverse estimation in Hilbert scales. SIAM J. Appl. Math. 56 1424-1444.

MARTEAU, C. and MathÉ, P. (2014). General regularization schemes for signal detection in inverse problems. Math. Methods Statist. 23 176-200.

Mathé, P. and Pereverzev, S. V. (2002). Direct estimation of linear functionals from indirect noisy observations. J. Complexity 18 500-516. MR1919446

MeIster, A. (2009). Deconvolution Problems in Nonparametric Statistics. Lecture Notes in Statistics 193. Springer, Berlin.

NATTERER, F. (1986). The Mathematics of Computerized Tomography. B. G. Teubner, Stuttgart.

NicKL, R. and REISs, M. (2012). A Donsker theorem for Lévy measures. J. Funct. Anal. 263 33063332 .

NIKOL'SKIĬ, S. M. (1951). Inequalities for entire functions of finite degree and their application in the theory of differentiable functions of several variables. In Trudy Mat. Inst. Steklov. 38 244-278. Izdat. Akad. Nauk SSSR, Moscow.

O'Sullivan, F. (1986). A statistical perspective on ill-posed inverse problems. Statist. Sci. $1502-$ 527.

PICKANDS, J. III (1969). Upcrossing probabilities for stationary Gaussian processes. Trans. Amer. Math. Soc. 145 51-73.

Piterbarg, V. I. (1996). Asymptotic Methods in the Theory of Gaussian Processes and Fields. Translations of Mathematical Monographs 148. Amer. Math. Soc., Providence, RI.

Proksch, K., Werner, F. and MunK, A. (2018). Supplement to "Multiscale scanning in inverse problems.” DOI:10.1214/17-AOS1669SUPP.

RAY, K. (2013). Bayesian inverse problems with non-conjugate priors. Electron. J. Stat. 7 25162549. 
RAY, K. (2017). Adaptive Bernstein-von Mises theorems in Gaussian white noise. Ann. Statist. 45 2511-2536. MR3737900

RIO, E. (1993). Strong approximation for set-indexed partial-sum processes, via KMT constructions. II. Ann. Probab. 21 1706-1727. MR1235436

RoHDE, A. (2008). Adaptive goodness-of-fit tests based on signed ranks. Ann. Statist. $361346-$ 1374. MR2418660

Rufibach, K. and Walther, G. (2010). The block criterion for multiscale inference about a density, with applications to other multiscale problems. J. Comput. Graph. Statist. 19 175-190.

Schmidt-Hieber, J., MunK, A. and DÜMbGen, L. (2013). Multiscale methods for shape constraints in deconvolution: Confidence statements for qualitative features. Ann. Statist. 41 12991328. MR3113812

Schwartzman, A., Dougherty, R. F. and TAYlor, J. E. (2008). False discovery rate analysis of brain diffusion direction maps. Ann. Appl. Stat. 2 153-175. MR2415598

SharpNACK, J. and ARIAS-CASTRO, E. (2016). Exact asymptotics for the scan statistic and fast alternatives. Electron. J. Stat. 10 2641-2684.

SöHL, J. and TRABS, M. (2012). A uniform central limit theorem and efficiency for deconvolution estimators. Electron. J. Stat. 6 2486-2518.

Ta, H., Keller, J., Haltmeier, M., Saka, S. K., Schmied, J., Opazo, F., Tinnefeld, P., MunK, A. and HELl, S. W. (2015). Mapping molecules in scanning far-field fluorescence nanoscopy. Nat. Commun. 67977.

TSYBAKOV, A. (2000). On the best rate of adaptive estimation in some inverse problems. C. R. Acad. Sci. Paris Sér. I Math. 330 835-840. MR1769957

TSYBAKOV, A. B. (2009). Introduction to Nonparametric Estimation. Springer, New York.

WALther, G. (2010). Optimal and fast detection of spatial clusters with scan statistics. Ann. Statist. 38 1010-1033. MR2604703

WILLER, T. (2009). Optimal bounds for inverse problems with Jacobi-type eigenfunctions. Statist. Sinica 19 785-800. MR2514188

K. PROKSCH

A. MUNK

INSTITUTE FOR MATHEMATICAL STOCHASTICS

UNIVERSITY OF GÖTTINGEN

GOLDSCHMIDTSTRASSE 7

37077 GÖTTINGEN

GERMANY

E-MAIL: kproksc@uni-goettingen.de munk@math.uni-goettingen.de

\author{
F. WERNER \\ MAX PLANCK InSTITUTE FOR \\ BIOPHYSICAL CHEMISTRY \\ AM FASSBERG 11 \\ 37077 GÖTTINGEN \\ GERMANY \\ E-MAIL: Frank.Werner@mpibpc.mpg.de
}

